Article

\title{
Exact Controllability Results for Sobolev-Type Hilfer Fractional Neutral Delay Volterra-Fredholm Integro-Differential Systems
}

\author{
Velusamy Vijayakumar ${ }^{1}$, Saud Fahad Aldosary ${ }^{2}$ and Kottakkaran Sooppy Nisar ${ }^{2, *(1)}$ \\ 1 Department of Mathematics, School of Advanced Sciences, Vellore Institute of Technology, \\ Vellore 632 014, Tamil Nadu, India; vijayakumarv@vit.ac.in \\ 2 Department of Mathematics, College of Arts and Sciences, Prince Sattam bin Abdulaziz University, \\ Wadi Aldawaser 11991, Saudi Arabia; sau.aldosary@psau.edu.sa \\ * Correspondence: n.sooppy@psau.edu.sa
}

check for updates

Citation: Vijayakumar, V.; Aldosary, S.F.; Nisar, K.S. Exact Controllability Results for Sobolev-Type Hilfer Fractional Neutral Delay Volterra-Fredholm

Integro-Differential Systems. Fractal Fract. 2022, 6, 81. https://doi.org/ 10.3390 / fractalfract6020081

Academic Editor: Stanislaw Migorski

Received: 16 December 2021

Accepted: 20 January 2022

Published: 1 February 2022

Publisher's Note: MDPI stays neutral with regard to jurisdictional claims in published maps and institutional affiliations.

Copyright: () 2022 by the authors Licensee MDPI, Basel, Switzerland. This article is an open access article distributed under the terms and conditions of the Creative Commons Attribution (CC BY) license (https:// creativecommons.org/licenses/by/ $4.0 /)$.

\begin{abstract}
This manuscript mainly focuses on the exact controllability of Sobolev-type Hilfer fractional neutral delay Volterra-Fredholm integro-differential systems. The principal findings of this discussion are established by using the theories on fractional calculus, the measure of noncompactness and Mönch fixed point technique. Initially, the exact controllability of the system is presented and then we improve the discussion to the system with nonlocal conditions. Finally, abstract and filter systems are provided for the illustration.
\end{abstract}

Keywords: controllability; Hilfer fractional system; neutral system; Volterra-Fredholm integrodifferential equations; nonlocal conditions; measure of noncompactness

\section{Introduction}

In many physical processes, fractional differential equations incorporating not only one fractional derivative but also several fractional derivatives are heavily concentrated. The meaning of fractional systems has recently attracted a lot of attention due to its astonishing applications in showcasing the wonders of science and engineering. The use of fractional order differential equations allows for the management of a wide range of issues in a variety of fields, including fluid flow, electrical systems, visco-elasticity, electro-chemistry, and so on. The monographs [1-9] and the research articles [8-13] show the interlinking in the same way that the separation between classical and fractional differential representations seems to. Applications of the differential systems can be found in [14-16]. Neutral structures with delays or without delays, in particular, serve as a summary association of a large number of partial neutral structures that appear in problems involving heat flow in substances, visco-elasticity, and a variety of natural processes. Neutral systems appear in many areas of applied mathematics; as a result, the most successful neutral structures have gotten a lot of attention in the current generation; readers can look at [12,13,17-21].

Recently, in [22,23], the author initiated another kind of derivative of fractional order, that including Riemann-Liouville and Caputo fractional derivative. In [24], the authors proved the existence of mild solution for evolution equation with Hilfer fractional derivative which generalized the famous Riemann-Liouville fractional derivative by using the semigroup theory, measure of noncompactness and fixed point approach. In [25], the authors proved the approximate controllability of Hilfer fractional neutral stochastic integro-differential systems by using fractional calculus and Bohenblust-Karlin's theorem. In $[18,26,27]$, the authors proved the existence and controallbility of various extensions related with Hilfer fractional derivative by using semigroup theory, measure of noncompactness and various fixed point theorems.

In [28], the authors discussed the approximate controllability of non-densely defined Sobolev-type Hilfer fractional neutral delay differential system by using BohenblustKarlin's fixed point theorem. In [29], the authors proved the existence of nonlocal functional 
integro-differential equations via Hilfer fractional derivative by using Mönch fixed point theorem. In [13], the authors discusssed the existence of Sobolev-type Hilfer fractional neutral integro-differential equations with infinite delay by uisng Mönch fixed point theorem. In [30], the authors proved the approximate controllability of Hilfer fractional differential inclusions with nonlocal conditions by using Bohenblust-Karlin's fixed point theorem. The existence and exact controllability described in our paper have still to be investigated, and it is the motivation of this article.

Motivated by the monograph, nowadays, several authors focus on this Hilfer fractional derivative, and we refer to [13,18,22,24-27,29-32]. The potential of controllability is an important part of engineering and mathematical control theory. Finding a suitable control function to the point where one may guide the considered dynamic system to a final state is the controllability problem. As a result, many researchers have investigated the controllability of a variety of nonlinear systems in recent years, and the articles are available for viewing. For instance, refs. $[12,13,18,19,30,32-45]$ and references therein.

Assume that the Hilfer fractional neutral delay Volterra-Fredholm integro-differential system of Sobolev-type has the following form

$$
\begin{aligned}
D_{0^{+}}^{v, \zeta}[J u(t)- & \left.F_{1}\left(t, u_{t}\right)\right]=A u(t)+B x(t) \\
& +F_{2}\left(t, u_{t}, \int_{0}^{t} e\left(t, \tau, u_{\tau}\right) d \tau, \int_{0}^{c} f\left(t, \tau, u_{\tau}\right) d \tau\right), t \in I=(0, c], \\
I_{0^{+}}^{(1-v)(1-\zeta)} u(0) & =\phi \in \mathcal{R}_{l},
\end{aligned}
$$

where $D_{0^{+}}^{v, \zeta}$ stands for the Hilfer fractional derivative, $0 \leq v \leq 1 ; \frac{1}{2}<\zeta<1$; and $x(\cdot)$ takes value in Banach Space $X$ with $\|\cdot\|$. The histories $u_{\varrho}:(-\infty, 0] \rightarrow \mathcal{R}_{l}, u_{\varrho}(s)=u(\varrho+s)$, $s \leq 0$ with phase space $\mathcal{R}_{l} . F_{1}: I \times \mathcal{R}_{l} \rightarrow X, F_{2}: I \times \mathcal{R}_{l} \times X \times X \rightarrow X, e: I \times I \times \mathcal{R}_{l} \rightarrow X$ and $f: I \times I \times \mathcal{R}_{l} \rightarrow X$ are appropriate functions. $u(\cdot)$ is a control function and $B$ is a bounded linear operator from $\mathscr{V} \rightarrow X$.

The rest of the paper is organized as follows:

1. Section 2: Theoretical notions linked to fractional calculus and the measure of noncompactness are presented.

2. Section 3: This section is focused on exact controllability results of the fractional system (1) and (2).

3. Section 4: Our findings are expanded to include the concept of nonlocal situations.

4. Section 5: Finally, abstract and filter systems are provided for the illustration of the obtained theory.

\section{Preliminaries}

We now provide some fundamental theories, lemmas, and facts to discuss our main results. $C(I, X)$ - the spaces of all continuous functions. Let us take $\eta=v+\zeta-v \zeta$, then $(1-\eta)=(1-v)(1-\zeta)$. Assume $C_{1-\eta}(I, X)=\left\{u: t^{1-\eta} u(t) \in C(I, X)\right\}$ with $\|\cdot\|_{\eta}$ by $\|u\|_{c}=\sup \left\{t^{1-\eta}\|u(t)\|, t \in I, \eta=(v+\zeta-v \zeta)\right\}$. Clearly, $C_{1-\eta}(I, X)$ is a Banach space. Define $H$ with $\|H\|_{L^{\rho}\left(I, \mathbb{R}^{+}\right)}$if $H \in L^{\rho}\left(I, \mathbb{R}^{+}\right)$for any $\rho$ with $1 \leq \rho \leq \infty$. $A^{\alpha}, 0<\alpha \leq 1$, a closed linear operator on $D\left(A^{\alpha}\right)$ with inverse $A^{-\alpha}$, see [46].

Definition 1 ([46]).

(i) $D\left(A^{\alpha}\right)$, a Banach space with $\|u\|_{\alpha}=\left\|A^{\alpha} u\right\|$ for $u \in D\left(A^{\alpha}\right)$.

(ii) $T(t): X \rightarrow X_{\alpha}$, for $t \geq 0$.

(iii) $A^{\alpha} T(t) u=T(t) A^{\alpha} u$, for $u \in D\left(A^{\alpha}\right)$ and $t \geq 0$.

(iv) $A^{\alpha} T(t)$ is bounded on $X$ and there exists $M_{\alpha}>0$ such that

$$
\left\|A^{\alpha} T(t)\right\| \leq \frac{M_{\alpha}}{t^{\alpha}}
$$


Definition 2 ([47]). The operators define $A: D(A) \subset X \rightarrow X$ and $J: D(A) \subset X \rightarrow X$ satisfy the following:

( $\left.\mathbf{J}_{1}\right)$ A and $J$ are closed linear operators.

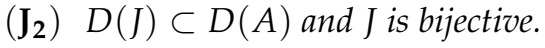

$\left(\mathbf{J}_{3}\right) J^{-1}: X \rightarrow D(J)$ is continuous.

Additionally, because of $\left(\mathbf{J}_{\mathbf{1}}\right)$ and $\left(\mathbf{J}_{\mathbf{2}}\right) \mathrm{J}^{-1}$ is closed, by $\left(\mathbf{J}_{\mathbf{3}}\right)$ and by referring closed graph theorem, we obtain the boundedness of $A J^{-1}: X \rightarrow X$. Define $\left\|J^{-1}\right\|=\widetilde{J}_{m}$ and $\|J\|=J_{m}$.

Definition 3 ([4]). The left sided Riemann-Liouville fractional integral of order $\zeta$ having lower limit $c$ for $F:[c,+\infty) \rightarrow \mathbb{R}$ is presented as

$$
I_{c^{+}}^{\zeta} F(\varrho)=\frac{1}{\Gamma(\zeta)} \int_{c}^{\varrho} \frac{F(\tau)}{(\varrho-\tau)^{1-\zeta}} d \tau, \varrho>c ; \zeta>0,
$$

if the right side is pointwise determined on $[c,+\infty)$, where $\Gamma(\cdot)$ denotes gamma function.

Definition 4 ([4]). The left-sided Riemann-Liouville fractional derivative of order $\zeta \in[k-1, k)$, $k \in X$ for $F:[c,+\infty) \rightarrow \mathbb{R}$ is given by

$$
{ }^{L} D^{\zeta}{ }_{c^{+}} F(\varrho)=\frac{1}{\Gamma(k-\zeta)} \frac{d^{k}}{d \varrho^{k}} \int_{c}^{\varrho} \frac{F(\tau)}{(\varrho-\tau)^{\zeta+1-k}} d \tau, \quad \varrho>c, k-1<\zeta<k .
$$

Definition 5 ([4]). The left-sided Hilfer fractional derivative of order $0 \leq v \leq 1$ and $0<\zeta<1$ function of $F(\varrho)$ is given by

$$
D_{c^{+}}^{v, \zeta} F(\varrho)=\left(I_{c^{+}}^{v(1-\zeta)} D\left(I_{d^{+}}^{(1-v)(1-\zeta)} F\right)\right)(\varrho)
$$

Remark 1 ([23]).

(i) Given $\zeta=0,0<v<1$ also $c=0$, the Hilfer fractional derivative identical with standard Riemann-Liouville fractional derivative:

$$
D_{0^{+}}^{0, \zeta} F(\varrho)=\frac{d}{d \varrho} I_{0^{+}}^{1-\zeta} F(\varrho)={ }^{L} D^{\zeta} 0^{+} F(\varrho)
$$

(ii) Given $\zeta=1,0<v<1$ also $c=0$, the Hilfer fractional derivative identical with standard Caputo derivative:

$$
D_{0^{+}}^{1, \zeta} F(\varrho)=I_{0^{+}}^{1-\zeta} \frac{d}{d \varrho} F(\varrho)={ }^{C} D^{\zeta} 0^{+} F(\varrho)
$$

We define the abstract phase space $\mathcal{R}_{l}$ by referring [33]. Consider $l:(-\infty, 0] \rightarrow$ $(0,+\infty)$ is continuous along with $j=\int_{-\infty}^{0} l(\gamma) d \gamma<+\infty$. Now for every $a>0$, we define

$$
\mathcal{R}_{l}=\{\eta:[-a, 0] \rightarrow X \text { such that } \eta(\gamma) \text { is bounded and measurable }\},
$$

and

$$
\|\eta\|_{[-a, 0]}=\sup _{\zeta \in[-a, 0]}\|\eta(\zeta)\|, \text { for all } \eta \in \mathcal{R}
$$

Now, we define

$$
\begin{gathered}
\mathcal{R}_{l}=\left\{\eta:(-\infty, 0] \rightarrow X \text { such that for some } c>0,\left.\eta\right|_{[-c, 0]} \in \mathcal{R}\right. \\
\text { and } \left.\int_{-\infty}^{0} l(\zeta)\|\eta\|_{[\zeta, 0]} d \zeta<+\infty\right\},
\end{gathered}
$$


and

$$
\|\eta\|_{\mathcal{R}_{l}}=\int_{-\infty}^{0} j(\zeta)\|\eta\|_{[\zeta, 0]} d \zeta, \forall \eta \in \mathcal{R}_{l},
$$

therefore $\left(\mathcal{R}_{l},\|\cdot\|_{\mathcal{R}_{l}}\right)$ is a Banach space.

Consider

$$
\mathcal{R}_{l}^{\prime}=\left\{u:(-\infty, c] \rightarrow X \text { such that }\left.u\right|_{V} \in C(I, X), u(0)=\phi(0) \in \mathcal{R}_{l}\right\} .
$$

Set $\|\cdot\|_{c}$ be a seminorm in $\mathcal{R}_{l}^{\prime}$ which is defined by

$$
\|u\|_{c}=\|\phi(0)\|_{\mathcal{B}_{h}}+\sup \{|y(\zeta)|: \zeta \in[0, c]\}, u \in \mathcal{R}_{l}^{\prime} .
$$

Lemma 1 ([48]). If $u \in \mathcal{R}_{l}^{\prime}$, then for $\gamma \in I, u_{\gamma} \in \mathcal{R}_{l}$. Furthermore,

$$
j\|u(\gamma)\| \leq\left\|u_{\gamma}\right\|_{\mathcal{R}_{l}} \leq\|\phi(0)\|_{\mathcal{R}_{l}}+l \sup _{\zeta \in[0, \gamma]}|u(\zeta)|,
$$

where $j=\int_{-\infty}^{0} l(\gamma) d \gamma<+\infty$.

Lemma 2. The function $u:[0, c] \rightarrow X$ is said to be an integral solution of the fractional system (1) and (2) if it satisfies the following:

- $u:[0, c] \rightarrow X$ is continuous.

- $I_{0^{+}}^{1-\zeta} u(t) \in D(A)$, for $t \in I$.

- $\quad$ The fractional system (1)-(2) is similar with

$$
\begin{aligned}
u(t)= & \frac{J^{-1}\left[\phi(0)-F_{1}(0, \phi(0))\right]}{\Gamma(v(1-\zeta)+\zeta)} J t^{(v-1)(1-\zeta)}+J^{-1} F_{1}\left(t, u_{t}\right) \\
& +\frac{1}{\Gamma(\zeta)} \int_{0}^{t}(t-s)^{\zeta-1} J^{-1}[A u(s)+B x(s) \\
& \left.+F_{2}\left(s, u_{s}, \int_{0}^{s} e\left(s, \tau, u_{\tau}\right) d \tau, \int_{0}^{c} f\left(s, \tau, u_{\tau}\right) d \tau\right)\right] d s, t \in I
\end{aligned}
$$

Remark 2. By referring Wright function $M_{\zeta}(s)$, we present the mild solution of the fractional system (1)-(2) is as follows:

$$
M_{\zeta}(s)=\sum_{k=1}^{\infty} \frac{(-s)^{k-1}}{(k-1) ! \Gamma(1-k \zeta)}, 0<\zeta<1, s \in \mathbb{C},
$$

and satisfies

$$
\int_{0}^{\infty} s^{s} M_{\zeta}(s) d s=\frac{\Gamma(1+s)}{\Gamma(1+\zeta s)}, \text { for } s \geq 0 .
$$

Lemma 3. If the fractional system (1)-(2) are satisfied, then there exists $F_{1}: I \times \mathcal{R}_{l} \rightarrow X$ and $F_{2}: I \times \mathcal{R}_{l} \times X \times X \rightarrow X$ such that

$$
\begin{aligned}
u(t)=J^{-1} & \mathscr{P}_{\nu, \zeta}(t) J\left(\phi(0)-F_{1}(0, \phi(0))+J^{-1} F_{1}\left(t, u_{t}\right)+\int_{0}^{t} J^{-1} \mathscr{R}_{\zeta}(t) A F_{1}\left(s, u_{s}\right) d s\right. \\
& +\int_{0}^{t} J^{-1} \mathscr{R}_{\zeta}(t) F_{2}\left(s, u_{s}, \int_{0}^{s} e\left(s, \tau, u_{\tau}\right) d \tau, \int_{0}^{c} f\left(s, \tau, u_{\tau}\right) d \tau\right) d s \\
& +\int_{0}^{t} J^{-1} \mathscr{R}_{\zeta}(t) B x(s) d s, \quad t \in I,
\end{aligned}
$$


where

$$
\mathscr{P}_{\nu, \zeta}(t)=I_{0^{+}}^{v(1-\zeta)}(t)^{\zeta-1} \mathscr{S}_{\zeta}(t) ; \mathscr{R}_{\zeta}(t)=t^{\zeta-1} \mathscr{S}_{\zeta}(t) ; \mathscr{S}_{\zeta}(t)=\int_{0}^{\infty} \zeta \omega M_{\zeta}(\omega) S\left(t^{\zeta} \omega\right) d \omega .
$$

Definition 6 ([20]). A function $u:(-\infty, c] \rightarrow X$ is said to be a mild solution of the fractional system (1)-(2) provided that $u_{0}=\phi(0) \in \mathcal{R}_{l}$ on $(-\infty, 0]$ and satisfies the following integral equation

$$
\begin{aligned}
u(t)= & J^{-1} \mathscr{P}_{v, \zeta}(t) J\left[\phi(0)-F_{1}(0, \phi(0))\right]+J^{-1} F_{1}\left(t, u_{t}\right) \\
& +\int_{0}^{t}(t-s)^{\zeta-1} J^{-1} \mathscr{S}_{\zeta}(t-s) A J^{-1} F_{1}\left(s, u_{s}\right) d s \\
& +\int_{0}^{t}(t-s)^{\zeta-1} J^{-1} \mathscr{S}_{\zeta}(t-s) F_{2}\left(s, u_{s}, \int_{0}^{s} e\left(s, \tau, u_{\tau}\right) d \tau, \int_{0}^{c} f\left(s, \tau, u_{\tau}\right) d \tau\right) d s \\
& +\int_{0}^{t}(t-s)^{\zeta-1} J^{-1} \mathscr{S}_{\zeta}(t-s) B x(s) d s, t \in I,
\end{aligned}
$$

where

$$
\mathscr{P}_{v, \zeta}(t)=\int_{0}^{\infty} \xi_{\zeta}(\omega) M\left(t^{\zeta} \omega\right) d \omega, \quad \mathscr{I}_{\zeta}=\zeta \int_{0}^{\infty} \omega \xi_{\zeta}(\omega) M\left(t^{\zeta} \omega\right) d \omega,
$$

and for $\omega \in(0, \infty)$

$$
\begin{gathered}
\xi_{\zeta}(\omega)=\frac{1}{\zeta} \omega^{-1-\frac{1}{\zeta} \bar{w}_{\zeta}}\left(\omega^{-\frac{1}{\zeta}}\right) \geq 0, \\
\bar{w}_{\zeta}(\omega)=\frac{1}{\pi} \sum_{n=1}^{\infty}(-1)^{n-1} t^{-n \zeta-1} \frac{\Gamma(n \zeta+1)}{n !} \sin (n \pi \zeta),
\end{gathered}
$$

where $\xi_{\zeta}$ is a probability density function defined on $(0, \infty)$, that is,

$$
\xi_{\zeta}(\omega) \geq 0, \quad \omega \in(0, \infty) \text { and } \int_{0}^{\infty} \xi_{\zeta}(\omega) d \omega=1 .
$$

Lemma 4 ([20]). The operators $\mathscr{P}_{v, \zeta}$ and $\mathscr{S}_{\zeta}$ satisfies the following:

(i) For $t \geq 0, \mathscr{P}_{v, \zeta}$ and $\mathscr{S}_{\zeta}$ are linear and bounded, that is, for every $u \in X$,

$$
\left\|\mathscr{P}_{v, \zeta}(t) u\right\| \leq \frac{M t^{\eta-1}}{\Gamma(v(1-\zeta)+\zeta)}\|u\| \text { and }\left\|\mathscr{S}_{\zeta}(t) u\right\| \leq \frac{M}{\Gamma(\zeta)}\|u\|,
$$

where $\mathscr{P}_{v, \zeta}(t)=I_{0^{+}}^{v(1-\zeta)} \mathscr{R}_{\zeta}(t), \mathscr{R}_{\zeta}(t)=t^{\zeta-1} \mathscr{S}_{\zeta}(t)$.

(ii) The operators $\left\{\mathscr{P}_{v, \zeta}(t)\right\}_{t \geq 0}$ and $\left\{\mathscr{S}_{\zeta}(t)\right\}_{\geq 0}$ are strongly continuous.

(iii) For every $u \in X, \mu, \zeta \in(0,1]$, we have

$$
\begin{gathered}
A \mathscr{S}_{\zeta}(t) u=A^{1-\mu} \mathscr{S}_{\zeta}(t) A^{\mu} u, 0 \leq t \leq c ; \\
\left\|A^{\mu} \mathscr{S}_{\zeta}(t)\right\| \leq \frac{\zeta C_{\mu} \Gamma(2-\mu)}{t^{\zeta \mu} \Gamma(1+\zeta(1-\mu))}, 0<t \leq c .
\end{gathered}
$$

Lemma 5. The operators $\left\{\mathscr{S}_{\zeta}(t)\right\}_{t>0}$ and $\left\{\mathscr{P}_{v, \zeta}(t)\right\}_{t>0}$ are strongly continuous, that is, $0<t^{\prime}<t^{\prime \prime} \leq c$,

$$
\left\|\left(t^{\prime}\right)^{\zeta-1} \mathscr{S}_{\zeta}\left(t^{\prime}\right) u-\left(t^{\prime \prime}\right)^{\zeta-1} \mathscr{S}_{\zeta}\left(t^{\prime \prime}\right) u\right\| \rightarrow 0 \text { and } \quad\left\|\mathscr{P}_{v, \zeta}\left(t^{\prime}\right) u-\mathscr{P}_{v, \zeta}\left(t^{\prime \prime}\right) u\right\| \rightarrow 0 \text { as } t^{\prime \prime} \rightarrow t^{\prime} .
$$

Definition $7([49,50])$. The Measure of noncompactness of Hausdorff $\mathscr{T}(\cdot)$ determined on every bounded subset $\varrho$ of $X$ by $\mathscr{T}(\varrho)=\inf \{\epsilon>0: \varrho$ can be covered by a finite number of balls of radii lesser than $\epsilon$. 
Definition 8 ([35]). Let $F^{+}$be the positive cone of an order Banach space $(F, \leq)$. The value E of $F^{+}$is said to be measure of noncompactness on X of $\mathscr{D}$ determined on the set of all bounded subsets of $X$ if and only if $E(\overline{c o} \varrho)=E(\varrho)$ for all bounded subsets $\varrho \subseteq X$, where $\overline{c o} \varrho$ is a closed convex hull of $\varrho$.

Definition 9 ([49,51]). For every bounded subsets $\varrho, \varrho_{1}, \varrho_{2}$ of X.

(i) Monotone if and only if for all bounded subsets $\varrho, \varrho_{1}, \varrho_{2}$ of $X$ we get: $\left(\varrho_{1} \subseteq \varrho_{2}\right) \Rightarrow\left(\mathscr{D}\left(\varrho_{1}\right) \leq\right.$ $\left.\mathscr{D}\left(\varrho_{2}\right)\right)$;

(ii) Non singular if and only if $\mathscr{D}(\{a\} \cup \varrho)=\mathscr{D}(\varrho)$ for each $a \in X, \varrho \subset U$;

(iii) Regular if and only if $\mathscr{D}(\varrho)=0$ if and only if $\varrho$ is relatively compact in $X$;

(iv) $\mathscr{T}\left(\varrho_{1}+\varrho_{2}\right) \leq \mathscr{T}\left(\varrho_{1}\right)+\mathscr{T}\left(\varrho_{2}\right)$, where $\varrho_{1}+\varrho_{2}=\left\{x_{1}+x_{2}: x_{1} \in \varrho_{1}, x_{2} \in \varrho_{2}\right\}$;

(v) $\mathscr{T}\left(\varrho_{1} \cup \varrho_{2}\right) \leq \max \left\{\mathscr{T}\left(\varrho_{1}\right), \mathscr{T}\left(\varrho_{2}\right)\right\}$;

(vi) $\mathscr{T}(\gamma \varrho) \leq|\gamma| \mathscr{T}(\varrho)$, for all $\gamma \in \mathbb{R}$;

(vii) If $Q: D(Q) \subseteq X \rightarrow Y$ is a Lipschitz continuous function with $k>0$, then $\mathscr{T}_{Y}(Q \varrho) \leq$ $k \mathscr{T}(\varrho)$, for $\varrho \subseteq D(Q)$ and $Y$ is a Banach space.

Lemma 6 ([49]). Assume that $\mathscr{X} \subset C([a, b], X)$ is bounded and equicontinuous, then $\mathscr{T}(\mathscr{X}(t))$ is continuous for any $t \in[a, b]$,

$$
\mathscr{T}(\mathscr{X})=\sup _{t \in N}\{\mathscr{T}(\mathscr{X}(t)), t \in[a, b]\}, \quad \text { whereby } \mathscr{X}(t)=\{u(t): u \in \mathscr{X}\} \subseteq X .
$$

Theorem 1 ([45,52]). Assume $\left\{u_{n}\right\}_{n=1}^{\infty}$ is a sequence of Bochner integrable functions from $I \rightarrow X$ with $\left\|u_{n}(t)\right\| \leq \varepsilon(t)$, for all $t \in I$ and every $n \geq 1$, where $\varepsilon \in L^{1}(I, \mathbb{R})$, then $\varrho(t)=\mathscr{T}\left(\left\{u_{n}(t)\right.\right.$ : $n \geq 1\}) \in L^{1}(I, \mathbb{R})$ and satisfies $\mathscr{T}\left\{\int_{0}^{t} \varrho(\tau) d \tau: n \geq 1\right\} \leq 2 \int_{0}^{t} \varrho(\tau) d \tau$.

Lemma 7 ([53]). Assume $F$ be closed convex subset of $X$ and $0 \in F, K: F \rightarrow X$ is continuous and that satisfies Mönch's condition, that is, $(P \subseteq F$ is countable, $P \subseteq \overline{c o}(\{0\} \cup K(P)) \Rightarrow \bar{P}$ is compact ). Then $K$ has a fixed point in $F$.

\section{Existence}

The reason for this part is to examine the existence of the fractional system (1)-(2).

$\left(\mathbf{H}_{0}\right)$ If $F \subset X$ and $w \in F$, then

$$
\left\|T\left(t_{2}^{v} \varrho\right) w-T\left(t_{1}^{v} \varrho\right) w\right\| \rightarrow 0, \text { when } t_{2} \rightarrow t_{1},
$$

for each fixed $\varrho \in(0, \infty)$.

$\left(\mathbf{H}_{1}\right)$ The function $F_{1}: I \times \mathcal{R}_{l} \rightarrow X$ is continuous and there exists $\mu \in(0,1)$ such that $F_{1} \in D\left(A^{\mu}\right)$, for every $w \in X, t \in I, A^{\mu} J^{-1} F_{1}(\cdot, w)$ is strongly measurable, there exists $M_{g}>0, M_{g}^{\prime}>0$ such that $r, s \in X, A^{\mu} F_{1}(t, \cdot)$ satisfies the following

$$
\begin{gathered}
\left\|A^{\mu} J^{-1} F_{1}(t, r(t))-A^{\mu} J^{-1} F_{1}(t, s(t)) \leq M_{g} t^{1-\eta}\right\| r(t)-s(t) \|_{\mathcal{R}_{l^{\prime}}} \\
\left\|A^{\mu} J^{-1} F_{1}(t, w(t))\right\| \leq M_{g}^{\prime}\left(1+t^{1-\eta}\|w\|_{\mathcal{R}_{l}}\right) .
\end{gathered}
$$

$\left(\mathbf{H}_{2}\right)$ The function $F_{2}: I \times \mathcal{R}_{l} \times X \times X \rightarrow X$ satisfies the following:

(i) The function $F_{2}(\cdot, \phi, y, z)$ is measurable for all $(\phi, y, z) \in \mathcal{R}_{l} \times X$ and $F_{2}(t, \cdot, \cdot, \cdot)$ is continuous for a.e. $t \in I, u \in \mathcal{R}_{l}, F_{2}(\cdot, \phi, y, z): I \rightarrow X$ is strongly measurable.

(ii) There exists $q_{1} \in(0, q)$ and $\epsilon_{1} \in L^{\frac{1}{q_{1}}}\left(I, \mathbb{R}^{+}\right)$and the integrable function $\kappa$ : $\mathbb{R}^{+} \rightarrow \mathbb{R}^{+}$such that $\|E(t, \phi, y, z)\| \leq \epsilon_{1}(t) \kappa\left(t^{1-\eta}\|\phi\|_{\mathcal{R}_{l}}+t^{1-\eta}\|y\|+t^{1-\eta}\|z\|\right)$, for all $(t, \phi, y, z) \in N \times \mathcal{R}_{l} \times X \times X$, where $\kappa$ satisfies $\lim _{n \rightarrow \infty} \inf \frac{\kappa(n)}{n}=0$. 
(iii) There exists $q_{2} \in(0, q)$ and $\epsilon_{2} \in L^{\frac{1}{q_{2}}}\left(I, \mathbb{R}^{+}\right)$such that for any bounded subset $D_{1} \subset X$ and $G_{1} \subset \mathcal{R}_{l}$,

$$
\mathscr{T}\left(F_{2}\left(t, G_{1}, D_{1}, D_{2}\right)\right) \leq \epsilon_{2}(t)\left[\sup _{-\infty<\varphi \leq 0} \mathscr{T}\left(G_{1}(\varphi)\right)+\mathscr{T}\left(D_{1}\right)+\mathscr{T}\left(D_{2}\right)\right],
$$

for a.e. $t \in I, G_{1}(\varrho)=\left\{v(\varrho): v \in G_{1}\right\}$ and $\mathscr{T}$ is the Hausdorff MNC.

$\left(\mathbf{H}_{3}\right)$ The function $e: I \times I \times \mathcal{R}_{l} \rightarrow X$ satisfies the following:

(i) $e(\cdot, \phi, z)$ is measurable for all $(\phi, z) \in \mathcal{R}_{l} \times X, f(t, \cdot, \cdot)$ is continuous for a.e. $t \in I$.

(ii) There exists $E_{0}>0$ such that $\|e(t, \tau, \phi)\| \leq E_{0}\left(1+\|\phi\|_{\mathcal{R}_{l}}\right)$, for all $t \in I, \tau \in X$, $\phi \in \mathcal{R}_{l}$.

(iii) There exists $q_{3} \in(0, q)$ and $\epsilon_{3} \in L^{\frac{1}{q_{3}}}\left(I, \mathbb{R}^{+}\right)$such that for any bounded subset $D_{3} \subset X$,

$$
\mathscr{T}\left(F_{2}\left(t, \tau, D_{3}\right)\right) \leq \epsilon_{3}(t, \tau)\left[\sup _{-\infty<t \leq 0} \mathscr{T}\left(D_{3}(\varphi)\right)\right] \text { for a.e. } t \in I,
$$

with $\epsilon_{3}^{*}=\sup _{\tau \in N} \int_{0}^{\tau} \epsilon_{3}(t, \varepsilon) d \varepsilon<\infty$.

$\left(\mathbf{H}_{4}\right)$ The function $f: I \times I \times \mathcal{R}_{l} \rightarrow X$ satisfies the following:

(i) $f(\cdot, \phi, z)$ is measurable for all $(\phi, z) \in \mathcal{R}_{l} \times X, f(t, \cdot, \cdot)$ is continuous for a.e. $t \in I$.

(ii) There exists $E_{1}>0$ such that $\|f(t, \tau, \phi)\| \leq E_{1}\left(1+\|\phi\|_{\mathcal{R}_{l}}\right)$, for all $t \in I, \tau \in X$, $\phi \in \mathcal{R}_{l}$.

(iii) There exists $q_{4} \in(0, q)$ and $\epsilon_{4} \in L^{\frac{1}{q_{4}}}\left(I, \mathbb{R}^{+}\right)$such that for any bounded subset $D_{4} \subset X$,

$$
\mathscr{T}\left(F_{2}\left(t, \tau, D_{4}\right)\right) \leq \epsilon_{4}(t, \tau)\left[\sup _{-\infty<t \leq 0} \mathscr{T}\left(D_{4}(\varphi)\right)\right] \text { for a.e. } t \in I,
$$

with $\epsilon_{4}^{*}=\sup _{\tau \in N} \int_{0}^{\tau} \epsilon_{4}(t, \varepsilon) d \varepsilon<\infty$.

$\left(\mathbf{H}_{\mathbf{5}}\right)$ The operator $B: L^{2}(I, \mathscr{V}) \rightarrow L^{1}(I, \mathscr{V})$ is bounded and $W: L^{2}(I, \mathscr{V}) \rightarrow L^{1}(I, \mathscr{V})$ is defined by

$$
W x=\int_{0}^{c}(c-s)^{\zeta-1} J^{-1} \mathscr{S}_{\zeta}(t-s) B x(s) d s,
$$

which satisfies the following:

(i) $W$ have an inverse $W^{-1}$ acquires the value in $L^{2}(I, \mathscr{V}) / \operatorname{Ker} W$, there exists $\epsilon_{b}>$ $0, \epsilon_{w}>0$ such that $\|B\| \leq \epsilon_{b}$ and $\left\|W^{-1}\right\| \leq \epsilon_{w}$.

(ii) For $q_{5} \in(0, q)$ and for every bounded subset $D \in X$, there exists $\epsilon_{4} \in L^{\frac{1}{q_{5}}}\left(I, \mathbb{R}^{+}\right)$ such that $\mathscr{R}\left(W^{-1}(D)(t)\right) \leq \epsilon_{5}(t) \mathscr{R}(D)$. Here $\epsilon_{n} \in L^{\frac{1}{q_{n}}}\left(I, \mathbb{R}^{+}\right)$.

(iii) For $q_{0} \in(0, q)$ and $M_{\mathscr{W}} \in L^{\frac{1}{q_{0}}\left(I, \mathbb{R}^{+}\right)}$such that for any $S \subset X, \mathscr{T}\left(\left(W^{-1} S\right)(t)\right) \leq$ $M_{\mathscr{W}}(t) \mathscr{T}(S), q_{n} \in(0, q), n=0,1,2,3$.

We present the following for our convenience:

$$
\begin{aligned}
& K_{1}=k_{1}\left\|\epsilon_{1}\right\|_{L^{\frac{1}{\eta_{1}}\left(I, \mathbb{R}^{+}\right)}}, \quad K_{2}=k_{2}\left\|\epsilon_{2}\right\|_{L^{\frac{1}{q_{2}}\left(I, \mathbb{R}^{+}\right)}}, \quad K_{3}=k_{3}\left\|\epsilon_{3}\right\|_{L^{\frac{1}{\eta_{3}}\left(I, \mathbb{R}^{+}\right)}}, \quad\left\|A^{-\mu}\right\|=M_{0}, \\
& K_{4}=k_{4}\left\|\epsilon_{4}\right\|_{L^{\frac{1}{q_{4}}\left(I, \mathbb{R}^{+}\right)}}, K_{5}=k_{5}\left\|\epsilon_{5}\right\|_{L^{\frac{1}{q_{5}}\left(I, \mathbb{R}^{+}\right)}}, K_{0}=k_{0}\left\|M_{\mathscr{W}}\right\|_{L^{\frac{1}{\eta_{0}}\left(I, \mathbb{R}^{+}\right)}} \\
& k_{n}=\left[\left(\frac{1-q_{n}}{\zeta-q_{n}}\right) c\left(\frac{\zeta-q_{n}}{1-q_{n}}\right)\right]^{1-q_{n}}, n=0,1,2,3,4, K=\frac{\zeta-1}{1-\rho^{\prime}}, \quad K^{*}=\frac{c^{(1+K)(1-\rho)}}{(1+K)^{1-\rho}} .
\end{aligned}
$$


Theorem 2. Suppose that the hypotheses $\left(\mathbf{H}_{\mathbf{0}}\right)-\left(\mathbf{H}_{\mathbf{5}}\right)$ are satisfied, then the fractional system (1) and (2) is controllable if

$$
L^{*}=\frac{2 M \widetilde{J}_{m} K_{2}\left(1+2 \epsilon_{3}^{*}+2 \epsilon_{4}^{*}\right) c^{1-\eta}}{\Gamma(\zeta)}\left[1+\frac{2 M \widetilde{J}_{m} K_{0} \epsilon_{c}}{\Gamma(\zeta)}\right], \text { for some } \frac{1}{2}<\zeta<1 .
$$

Proof. We now define the operator $\Phi: \mathcal{R}_{l}^{\prime} \rightarrow \mathcal{R}_{l}^{\prime}$ by

$$
\Phi u(t)=\left\{\begin{array}{l}
\phi(t), \quad t \in(-\infty, 0] \\
J^{-1} \mathscr{P}_{v, \zeta}(t) J\left[\phi(0)-F_{1}(0, \phi(0))\right]+J^{-1} F_{1}\left(t, u_{t}\right) \\
+\int_{0}^{t}(t-s)^{\zeta-1} J^{-1} A \mathscr{S}_{\zeta}(t-s) J^{-1} F_{1}\left(s, u_{s}\right) d s \\
+\int_{0}^{t}(t-s)^{\zeta-1} J^{-1} \mathscr{S}_{\zeta}(t-s) F_{2}\left(s, u_{s}, \int_{0}^{s} e\left(s, \tau, u_{\tau}\right) d \tau, \int_{0}^{c} f\left(s, \tau, u_{\tau}\right) d \tau\right) d s \\
+\int_{0}^{t}(t-s)^{\zeta-1} J^{-1} \mathscr{S}_{\zeta}(t-s) B x(s) d s, t \in I .
\end{array}\right.
$$

For $\phi \in \mathcal{R}_{l}$, we present $\hat{\beta}$ as follows:

$$
\hat{\beta}(t)=\left\{\begin{array}{l}
\phi(t), \quad t \in(-\infty, 0] \\
\mathscr{P}_{v, \zeta}(t) \phi(0), \quad t \in I
\end{array}\right.
$$

then $\hat{\beta} \in \mathcal{R}_{l}^{\prime}$. Let $u(t)=p(t)+\hat{\beta}(t),-\infty<t \leq d$. Clearly $u$ satisfies (3), if and only if $p$ satisfies $p_{0}=0$ and

$$
\begin{aligned}
p(t)= & -J^{-1} \mathscr{P}_{v, \zeta}(t) J F_{1}(0, \phi(0))+J^{-1} F_{1}\left(t, p_{t}+\hat{\beta}_{t}\right) \\
& +\int_{0}^{t}(t-s)^{\zeta-1} J^{-1} A \mathscr{S}_{\zeta}(t-s) J^{-1} F_{1}\left(s, p_{s}+\hat{\beta}_{s}\right) d s \\
& +\int_{0}^{t}(t-s)^{\zeta-1} J^{-1} \mathscr{S}_{\zeta}(t-s) B x_{p}(s) d s \\
& +\int_{0}^{t}(t-s)^{\zeta-1} J^{-1} \mathscr{S}_{\zeta}(t-s) \\
& (\times) F_{2}\left(s, p_{s}+\hat{\beta}_{s}, \int_{0}^{s} e\left(s, \tau, p_{\tau}+\hat{\beta}_{\tau}\right) d \tau, \int_{0}^{c} f\left(s, \tau, p_{\tau}+\hat{\beta}_{\tau}\right) d \tau\right) d s .
\end{aligned}
$$

where

$$
\begin{aligned}
& x_{p}(t)=W^{-1}\left[u^{1}-J^{-1} \mathscr{P}_{\nu, \zeta}(c) J\left[\phi(0)-F_{1}(0, \phi(0))\right]-J^{-1} F_{1}\left(c, u_{c}\right)\right. \\
& \quad-\int_{0}^{c}(c-s)^{\zeta-1} J^{-1} A \mathscr{S}_{\zeta}(c-s) J^{-1} F_{1}\left(s, u_{s}\right) d s \\
& \left.\quad+\int_{0}^{c}(c-s)^{\zeta-1} J^{-1} \mathscr{S}_{\zeta}(c-s) F_{2}\left(s, u_{s}, \int_{0}^{s} e\left(s, \tau, u_{\tau}\right) d \tau, \int_{0}^{c} f\left(s, \tau, u_{\tau}\right) d \tau\right) d s\right](t) .
\end{aligned}
$$

Take $\mathcal{R}_{l}^{\prime \prime}=\left\{p \in \mathcal{R}_{l}^{\prime}: p_{0}=0 \in \mathcal{R}_{l}\right\}$. For every $p \in \mathcal{R}_{l}^{\prime \prime}$,

$$
\begin{aligned}
\|p\|_{c} & =\left\|p_{0}\right\|_{\mathcal{R}_{l}}+\sup \{\|p(s)\|: 0 \leq s \leq c\}, \\
& =\sup \{\|p(s)\|: 0 \leq s \leq c\} .
\end{aligned}
$$

Hence $\left(\mathcal{R}_{l}^{\prime \prime},\|\cdot\|_{c}\right)$ is a Banach space. For $r>0$, fix $F_{r}=\left\{p \in \mathcal{R}_{l}^{\prime \prime}:\|w\|_{c} \leq r\right\}$, thus $F_{r} \subseteq \mathcal{R}_{l}^{\prime \prime}$ is uniformly bounded, and for $p \in F_{r}$, by referring Lemma 1 , we have

$$
\begin{aligned}
\left\|p_{t}+\hat{\beta}_{t}\right\|_{\mathcal{R}_{l}} & \leq\left\|p_{t}\right\|_{\mathcal{R}_{l}}+\left\|\hat{\beta}_{t}\right\|_{\mathcal{R}_{l}} \\
& \leq j\left(r+\frac{M}{\Gamma(v(1-\zeta)+\zeta)}|\phi(0)|\right)+\|\phi\|_{\mathcal{R}_{l}}=r^{\prime}
\end{aligned}
$$


Introduce $\tilde{Y}: \mathcal{R}_{l}^{\prime \prime} \rightarrow \mathcal{R}_{l}^{\prime \prime}$ by

$$
\widetilde{Y} p(t)=\left\{\begin{array}{l}
0, \quad t \in(-\infty, 0] \\
-\mathscr{P}_{v, \zeta}(t) F_{1}(0, \phi)+J^{-1} F_{1}\left(t, p_{t}+\hat{\beta}_{t}\right) \\
+\int_{0}^{t}(t-s)^{\zeta-1} J^{-1} A \mathscr{S}_{\zeta}(t-s) J^{-1} F_{1}\left(s, p_{s}+\hat{\beta}_{s}\right) d s \\
+\int_{0}^{t}(t-s)^{\zeta-1} J^{-1} \mathscr{S}_{\zeta}(t-s) B x_{p}(s) d s \\
+\int_{0}^{t}(t-s)^{\zeta-1} J^{-1} \mathscr{S}_{\zeta}(t-s) \\
\quad(\times) F_{2}\left(s, p_{s}+\hat{\beta}_{s}, \int_{0}^{s} e\left(s, \tau, p_{\tau}+\hat{\beta}_{\tau}\right) d \tau, \int_{0}^{c} f\left(s, \tau, p_{\tau}+\hat{\beta}_{\tau}\right) d \tau\right) d s .
\end{array}\right.
$$

Clearly, Y having a fixed point and which is similar to $\widetilde{Y}$. To prove $\widetilde{Y}$ having a fixed point, we subdivide the whole proof as follows:

Step 1: We state that there exists $r>0$ such that $\widetilde{Y}\left(F_{r}\right) \subseteq F_{r}$.

If it is not correct, then there exists $p^{r}(\cdot) \in F_{r}$ and $t \in I$ such that $\left\|\widetilde{\mathrm{Y}}\left(p^{r}\right)(t)\right\|>r$, i.e., $\widetilde{\mathrm{Y}}\left(p^{r}\right) \notin F_{r}$.

Fix $r>0$, and assume $\left\{F_{r}=u \in C:\|u\|_{c} \leq r\right\}$. Clearly, $F_{r}$ is a closed, bounded and convex set of $C$.

Now, we need to check there exists $r>0$ such that $\phi\left(F_{r}\right) \subseteq F_{r}$. If it fails, then $p^{r} \in F_{r}$. However, $\phi\left(u^{r}\right) \notin F_{r}$. Hence,

$$
\left\|\widetilde{Y}\left(u^{r}\right)\right\|_{c} \equiv \sup \left\{t^{1-\eta}\left\|s^{r}\right\|_{c}: s^{r} \in \phi\left(u^{r}\right)>r\right\} .
$$

Using assumptions $\left(\mathbf{H}_{\mathbf{2}}\right)$ and $\left(\mathbf{H}_{3}\right)$ and Lemma 4 , we have

$$
\begin{aligned}
r< & \sup _{t \in I} t^{1-\eta}\left\|\left(\widetilde{Y} p^{r}\right)(t)\right\| \\
\leq & c^{1-\eta}\left(\left\|-J^{-1} \mathscr{P}_{v, \zeta}(t) J F_{1}(0, \phi(0))\right\|+\left\|J^{-1} F_{1}\left(t, p_{t}+\hat{\beta}_{t}\right)\right\|\right. \\
& +\left\|\int_{0}^{t}(t-s)^{\zeta-1} J^{-1} A \mathscr{S}_{\zeta}(t-s) J^{-1} F_{1}\left(s, p_{s}+\hat{\beta}_{s}\right) d s\right\| \\
& +\left\|\int_{0}^{t}(t-s)^{\zeta-1} J^{-1} \mathscr{S}_{\zeta}(t-s) B x_{p}(s) d s\right\| \\
& +\| \int_{0}^{t}(t-s)^{\zeta-1} J^{-1} \mathscr{S}_{\zeta}(t-s) \\
& \left.(\times) F_{2}\left(s, p_{s}+\hat{\beta}_{s}, \int_{0}^{s} e\left(s, \tau, p_{\tau}+\hat{\beta}_{\tau}\right) d \tau, \int_{0}^{c} f\left(s, \tau, p_{\tau}+\hat{\beta}_{\tau}\right) d \tau\right) d s \|\right) \\
= & \sum_{i=1}^{5} V_{i},
\end{aligned}
$$

where

$$
\begin{aligned}
V_{1} & =c^{1-\eta}\left\|J^{-1} \mathscr{P}_{v, \zeta}(t) J F_{1}(0, \phi(0))\right\| \\
& \leq \frac{M_{0} M \widetilde{J}_{m} J_{m}}{\Gamma(v(1-\zeta)+\zeta)} M_{g^{\prime}}^{\prime} \\
V_{2} & =c^{1-\eta}\left\|J^{-1} F_{1}\left(t, p_{t}+\hat{\beta}_{t}\right)\right\| \\
& \leq c^{1-\eta} M_{0} \widetilde{J}_{m} M_{g}^{\prime}\left(1+r^{\prime}\right) .
\end{aligned}
$$

By referring the Hölder's inequality and Lemma 4, we have

$$
V_{3}=c^{1-\eta} \int_{0}^{t}\left\|(t-s)^{\zeta-1} J^{-1} A^{1-\zeta} \mathscr{S}_{\zeta}(t-s) A^{\zeta} J^{-1} F_{1}\left(s, p_{s}+\hat{\beta}_{s}\right)\right\| d s
$$




$$
\leq c^{1-\eta+\mu \zeta} \frac{C_{1-\mu} \Gamma(1+\mu)}{\mu \Gamma(1+\mu \zeta)} \widetilde{J}_{m} M_{g}^{\prime}\left(1+r^{\prime}\right) .
$$

By referring the Hypotheses $\left(\mathbf{H}_{\mathbf{1}}\right)-\left(\mathbf{H}_{\mathbf{5}}\right)$, we get

$$
\begin{aligned}
V_{4}= & c^{1-\eta} \| \int_{0}^{t}(t-s)^{\zeta-1} J^{-1} \mathscr{S}_{\zeta}(t-s) \\
& (\times) F_{2}\left(s, p_{s}+\hat{\beta}_{s}, \int_{0}^{s} e\left(s, \tau, p_{\tau}+\hat{\beta}_{\tau}\right) d \tau, \int_{0}^{c} f\left(s, \tau, p_{\tau}+\hat{\beta}_{\tau}\right) d \tau\right) d s \|, \\
\leq & c^{1-\eta} \widetilde{J}_{m}\left\|\int_{0}^{t}(t-s)^{\zeta-1} \mathscr{S}_{\zeta}(t-s) \epsilon_{1}(s) \mathrm{Y}\left(r^{\prime}+c E_{0}\left(1+r^{\prime}\right)+c E_{1}\left(1+r^{\prime}\right)\right) d s\right\| \\
\leq & c^{1-\eta} \frac{M \widetilde{J}_{m}}{\Gamma(\zeta)} K_{1} Y\left(r^{\prime}+c E_{0}\left(1+r^{\prime}\right)+c E_{1}\left(1+r^{\prime}\right)\right),
\end{aligned}
$$

and

$$
\begin{aligned}
& V_{5}= c^{1-\eta}\left\|\int_{0}^{t}(t-s)^{\zeta-1} J^{-1} \mathscr{S}_{\zeta}(t-s) B x_{p}(s) d s\right\|, \\
& \leq c^{1-\eta} \| \int_{0}^{t}(t-s)^{\zeta-1} J^{-1} \mathscr{S}_{\zeta}(t-s) B W^{-1}\left[u^{1}-J^{-1} \mathscr{P}_{v, \zeta}(c) J\left[\phi(0)-F_{1}(0, \phi)\right]\right. \\
&+J^{-1} F_{1}\left(c, p_{c}+\hat{\beta}_{c}\right)+\int_{0}^{c}(c-s)^{\zeta-1} J^{-1} A \mathscr{S}_{\zeta}(c-s) J^{-1} F_{1}\left(s, p_{s}+\hat{\beta}_{s}\right) d s \\
&+\int_{0}^{c}(c-s)^{\zeta-1} J^{-1} \mathscr{S}_{\zeta}(c-s) \\
&\left.\quad(\times) F_{2}\left(s, p_{s}+\hat{\beta}_{s}, \int_{0}^{s} e\left(s, \tau, p_{\tau}+\hat{\beta}_{\tau}\right) d \tau, \int_{0}^{c} f\left(s, \tau, p_{\tau}+\hat{\beta}_{\tau}\right) d \tau\right) d s\right] d s \| \\
& \leq c^{1-\eta+\zeta} \frac{M \widetilde{J}_{m} \epsilon_{b} \epsilon_{w}}{\Gamma(\eta)}\left[u^{1}+\frac{M \widetilde{J}_{m} J_{m} c^{\eta-1}}{\Gamma(s(1-\eta)+\eta)} M_{0}\left(\|\phi(0)\|+K_{h}^{\prime}\right)\right. \\
& \quad+M_{0} \widetilde{J}_{m} M_{g}^{\prime}\left(1+r^{\prime}\right)+c^{\mu \zeta} \frac{C_{1-\mu} \Gamma(1+\mu)}{\mu \Gamma(1+\mu \zeta)} \widetilde{J}_{m} M_{g}^{\prime}\left(1+r^{\prime}\right) \\
&\left.\quad+\frac{M \widetilde{J}_{m}}{\Gamma(\zeta)} K_{1} Y\left(r^{\prime}+c E_{0}\left(1+r^{\prime}\right)+c E_{1}\left(1+r^{\prime}\right)\right)\right] .
\end{aligned}
$$

Combining all the above results $V_{1}-V_{5}$, we get

$$
\begin{aligned}
r \leq & \frac{M_{0} M \widetilde{J}_{m} J_{m}}{\Gamma(v(1-\zeta)+\zeta)} M_{g}^{\prime}+c^{1-\eta} M_{0} \widetilde{J}_{m} M_{g}^{\prime}\left(1+r^{\prime}\right)+c^{1-\eta+\mu \zeta} \frac{C_{1-\mu} \Gamma(1+\mu)}{\mu \Gamma(1+\mu \zeta)} \widetilde{J}_{m} M_{g}^{\prime}\left(1+r^{\prime}\right) \\
& +c^{1-\eta} \frac{M \widetilde{J}_{m}}{\Gamma(\zeta)} K_{1} Y\left(r^{\prime}+c E_{0}\left(1+r^{\prime}\right)+c E_{1}\left(1+r^{\prime}\right)\right) \\
& +c^{1-\eta+\zeta} \frac{M \widetilde{J}_{m} \epsilon_{b} \epsilon_{w}}{\Gamma(\eta)}\left[u^{1}+\frac{M_{0} M \widetilde{J}_{m} J_{m} c^{\eta-1}}{\Gamma(v(1-\zeta)+\zeta)} M_{0}\|\phi(0)\|\right. \\
& +\frac{M_{0} M \widetilde{J}_{m} J_{m} c^{\eta-1}}{\Gamma(v(1-\zeta)+\zeta)} M_{0} K_{h}^{\prime}+M_{0} \widetilde{J}_{m} M_{g}^{\prime}\left(1+r^{\prime}\right)+c^{\mu \zeta} \frac{C_{1-\mu} \Gamma(1+\mu)}{\mu \Gamma(1+\mu \zeta)} \widetilde{J}_{m} M_{g}^{\prime}\left(1+r^{\prime}\right) \\
& \left.+\frac{M \widetilde{J}_{m}}{\Gamma(\zeta)} K_{1} Y\left(r^{\prime}+c E_{0}\left(1+r^{\prime}\right)+c E_{1}\left(1+r^{\prime}\right)\right)\right] \\
\leq & c^{1-\eta+\zeta} \frac{M \widetilde{J}_{m} \epsilon_{b} \epsilon_{w}}{\Gamma(\eta)}\left[u^{1}+\frac{M \widetilde{J}_{m} J_{m} c^{\eta-1}}{\Gamma(s(1-\eta)+\eta)} M_{0}\|\phi(0)\|\right] \\
& +\left[1+c^{\zeta} \frac{M \widetilde{J}_{m} \epsilon_{b} \epsilon_{w}}{\Gamma(\zeta)}\right]\left[\frac{M_{0} M \widetilde{J}_{m} J_{m}}{\Gamma(v(1-\zeta)+\zeta)} M_{g}^{\prime}+c^{1-\eta} M_{0} \widetilde{J}_{m} M_{g}^{\prime}\left(1+r^{\prime}\right)\right.
\end{aligned}
$$




$$
\begin{aligned}
& +c^{1-\eta+\mu \zeta} \frac{C_{1-\mu} \Gamma(1+\zeta)}{\zeta \Gamma(1+\mu \zeta)} \widetilde{J}_{m} M_{g}^{\prime}\left(1+r^{\prime}\right) \\
& \left.+c^{1-\eta} \frac{M \widetilde{J}_{m}}{\Gamma(\zeta)} K_{1} Y\left(r^{\prime}+c E_{0}\left(1+r^{\prime}\right)+c E_{1}\left(1+r^{\prime}\right)\right)\right] .
\end{aligned}
$$

If we divide (6) by $r$, and assuming $r \rightarrow \infty$, we have $1 \leq 0$, and this contradicts with(4), then, $\widetilde{\phi}\left(F_{r}\right) \subseteq F_{r}$.

Step 2: $\widetilde{Y}$ is continuous on $F_{r}$.

For $\widetilde{\phi}$ maps $F_{r}(I)$ into itself. For any $\left(p^{n}+\hat{\beta}\right), p, \hat{\beta} \in F_{r}(I), n=0,1,2, \cdots$, with $\lim _{n \rightarrow \infty} p^{n}+\hat{\beta}=(p+\hat{\beta})$, we have $\lim _{n \rightarrow \infty}\left(p^{n}+\hat{\beta}\right)(t)=(p+\hat{\beta})(t)$ and

$$
\lim _{n \rightarrow \infty} t^{1-\eta}\left(p^{n}+\hat{\beta}\right)=t^{1-\eta}(p+\hat{\beta}) .
$$

Assume $u(t)=t^{1-\eta}(p+\hat{\beta})$, then $\left\{\left(p^{n}+\hat{\beta}\right)\right\}_{n \in N} \subset F_{r}$ with $\left(p^{n}+\hat{\beta}\right) \rightarrow(p+\hat{\beta})$ in $F_{r}$ when $n \rightarrow \infty$. Define

$$
\begin{aligned}
& F_{2}\left(t, u_{n}(t), \int_{0}^{t} e\left(t, \tau, \tau^{1-\eta} u^{n}(\tau)\right) d \tau, \int_{0}^{c} f\left(t, \tau, \tau^{1-\eta} u^{n}(\tau)\right) d \tau\right) \\
& =F_{2}\left(t, t^{1-\eta}\left(p^{n}+\hat{\beta}\right)(t), \int_{0}^{t} e\left(t, \tau, \tau^{1-\eta}\left(p^{n}+\hat{\beta}\right)(\tau)\right) d \tau,\right. \\
& \left.\quad \int_{0}^{c} f\left(t, \tau, \tau^{1-\eta}\left(p^{n}+\hat{\beta}\right)(\tau)\right) d \tau\right) \\
& \rightarrow F_{2}\left(t, t^{1-\eta}(p+\hat{\beta})(t), \int_{0}^{t} e\left(t, \tau, \tau^{1-\eta}(p+\hat{\beta})(\tau)\right) d \tau,\right. \\
& \left.\qquad \int_{0}^{c} f\left(t, \tau, \tau^{1-\eta}(p+\hat{\beta})(\tau)\right) d \tau\right) \\
& =F_{2}\left(t, u(t), \int_{0}^{t} e\left(t, \tau, \tau^{1-\eta} u(\tau)\right) d \tau, \int_{0}^{c} f\left(t, \tau, \tau^{1-\eta} u(\tau)\right) d \tau\right), \text { as } n \rightarrow \infty,
\end{aligned}
$$

where

$$
\begin{aligned}
& \mathcal{F}_{n}(s)=F_{2}\left(s, s^{1-\eta}\left(p^{n}+\hat{\beta}\right)_{s}, \int_{0}^{s} e\left(s, \tau, \tau^{1-\eta}\left(p^{n}+\hat{\beta}\right)_{\tau}\right) d \tau,\right. \\
& \left.\qquad \int_{0}^{c} f\left(s, \tau, \tau^{1-\eta}\left(p^{n}+\hat{\beta}\right)_{\tau}\right) d \tau\right) \text { and } \\
& \mathcal{F}(s)=F_{2}\left(s, s^{1-\eta}(p+\hat{\beta})_{s}, \int_{0}^{s} e\left(s, \tau, \tau^{1-\eta}(p+\hat{\beta})_{\tau}\right) d \tau,\right. \\
& \left.\int_{0}^{c} f\left(s, \tau, \tau^{1-\eta}(p+\hat{\beta})_{\tau}\right) d \tau\right) .
\end{aligned}
$$

By referring $\left(\mathbf{H}_{\mathbf{1}}\right)$ and Lebesgue's dominated convergence theorem, we have

$$
\int_{0}^{t}(t-s)^{\zeta-1}\left\|J^{-1} \mathcal{F}_{n}(s)-J^{-1} \mathcal{F}(s)\right\| d s \rightarrow 0 \text { as } n \rightarrow \infty, t \in I .
$$

In view of $\left(\mathbf{H}_{\mathbf{1}}\right)$,

$$
\left\|\widetilde{\mathrm{Y}} p^{n}-\widetilde{\mathrm{Y}} p\right\|_{L^{\rho}} \leq \frac{M \widetilde{J}_{m}}{\Gamma(\zeta)} \int_{0}^{t}(t-s)^{\zeta-1}\left\|\mathcal{F}_{n}(s)-\mathcal{F}(s)\right\| d s+\frac{M \widetilde{J}_{m} \epsilon_{c} L^{*}}{\Gamma(\zeta)}\left\|x_{p^{n}}-x_{p}\right\|_{L^{\rho}},
$$


where

$$
\begin{aligned}
\left\|x_{p^{n}}-x_{p}\right\|_{L^{\rho}}= & W^{-1}\left[\int_{0}^{c}(c-s)^{\zeta-1} J^{-1} \mathscr{S}_{\zeta}(c-s)\left[\mathcal{F}_{n}(s)-\mathcal{F}(s)\right] d s\right](t) \\
& \leq \frac{M \widetilde{J}_{m} \epsilon_{w}}{\Gamma(\zeta)} \int_{0}^{c}(c-s)^{\zeta-1}\left[\mathcal{F}_{n}(s)-\mathcal{F}(s)\right] d s
\end{aligned}
$$

By using (7) and (8), we have

$$
\left\|\widetilde{Y} p^{n}-\widetilde{Y} p\right\|_{L^{\rho}} \rightarrow 0 \text { when } n \rightarrow \infty .
$$

Therefore $\widetilde{Y}$ is continuous on $F_{r}$.

Step 3: For $p \in F_{r}$, assume $p(t)=t^{\eta-1} u(t), \widetilde{Y}$ sends bounded sets into equicontinuous sets of $C$, for all $z \in F_{r}$, there exists $z \in \widetilde{Y}(z)$ such that $\left\|z\left(t_{2}\right)-z\left(t_{1}\right)\right\| \rightarrow 0$ when $t_{2} \rightarrow t_{1}$.

$$
\begin{aligned}
z(t)= & J^{-1} \mathscr{P}_{v, \zeta}(t) J\left[\phi(0)-F_{1}(0, \phi(0))\right]+J^{-1} F_{1}\left(t, p_{t}+\hat{\beta}_{t}\right) \\
& +\int_{0}^{t}(t-s)^{\zeta-1} J^{-1} \mathscr{S}_{\zeta}(t-s) A J^{-1} F_{1}\left(s, p_{s}+\hat{\beta}_{s}\right) d s \\
& +\int_{0}^{t}(t-s)^{\zeta-1} J^{-1} \mathscr{S}_{\zeta}(t-s) \\
& \quad(\times) F_{2}\left(s, p_{s}+\hat{\beta}_{s}, \int_{0}^{s} e\left(s, \tau, p_{\tau}+\hat{\beta}_{\tau}\right) d \tau, \int_{0}^{c} f\left(s, \tau, p_{\tau}+\hat{\beta}_{\tau}\right) d \tau\right) d s \\
& +\int_{0}^{t}(t-s)^{\zeta-1} J^{-1} \mathscr{S}_{\zeta}(t-s) B x_{p}(s) d s .
\end{aligned}
$$

Assume $0<\delta<t$ and $0<t_{1}<t_{2}<c$. Then $\widetilde{Y}\left(F_{r}\right)$ is equicontinuous on $I$.

$$
\begin{aligned}
\left\|z\left(t_{2}\right)-z\left(t_{1}\right)\right\| \\
=\| t_{2}^{1-\eta} J^{-1} \mathscr{P}_{\nu, \zeta}\left(t_{2}\right) J^{-1}\left[\phi(0)-F_{1}(0, \phi(0))\right] \\
\quad-t_{1}^{1-\eta} J^{-1} \mathscr{P}_{v, \zeta}\left(t_{1}\right) J^{-1}\left[\phi(0)-F_{1}(0, \phi(0))\right] \| \\
+\left\|A^{-\mu} J^{-1}\left[t_{2}^{1-\eta} A^{\mu} F_{1}\left(t_{2}, w_{t_{2}}+\hat{\beta}_{t_{2}}\right)-t_{1}^{1-\eta} A^{\mu} F_{1}\left(t_{1}, w_{t_{1}}+\hat{\beta}_{t_{1}}\right)\right]\right\| \\
+\| t_{2}^{1-\eta} \int_{0}^{t_{2}}\left(t_{2}-s\right)^{\zeta-1} J^{-1} A^{1-\mu} \mathscr{S}_{\zeta}\left(t_{2}-s\right) A^{\mu} J^{-1} F_{1}\left(s, w_{s}+\hat{\beta}_{s}\right) d s \\
\quad-t_{1}^{1-\eta} \int_{0}^{t_{1}}\left(t_{1}-s\right)^{\zeta-1} J^{-1} A^{1-\mu} \mathscr{S}_{\zeta}\left(t_{1}-s\right) A^{\mu} J^{-1} F_{1}\left(s, w_{s}+\hat{\beta}_{s}\right) d s \| \\
+\| t_{2}^{1-\eta} \int_{0}^{t_{2}}\left(t_{2}-s\right)^{\zeta-1} J^{-1} \mathscr{S}_{\zeta}\left(t_{2}-s\right) \\
\quad(\times) F_{2}\left(s, p_{s}+\hat{\beta}_{s}, \int_{0}^{s} e\left(s, \tau, p_{\tau}+\hat{\beta}_{\tau}\right) d \tau, \int_{0}^{c} f\left(s, \tau, p_{\tau}+\hat{\beta}_{\tau}\right) d \tau\right) d s \\
\quad-t_{1}^{1-\eta} \int_{0}^{t_{1}}\left(t_{1}-s\right)^{\zeta-1} J^{-1} \mathscr{S}_{\zeta}\left(t_{1}-s\right) \\
\quad(\times) F_{2}\left(s, p_{s}+\hat{\beta}_{s}, \int_{0}^{s} e\left(s, \tau, p_{\tau}+\hat{\beta}_{\tau}\right) d \tau, \int_{0}^{c} f\left(s, \tau, p_{\tau}+\hat{\beta}_{\tau}\right) d \tau\right) d s \| \\
\quad+\| t_{2}^{1-\eta} \int_{0}^{t_{2}}\left(t_{2}-s\right)^{\zeta-1} J^{-1} \mathscr{S}_{\zeta}\left(t_{2}-s\right) B x_{p}(s) d s \\
\quad-t_{1}^{1-\eta} \int_{0}^{t_{1}}\left(t_{1}-s\right)^{\zeta-1} J^{-1} \mathscr{S}_{\zeta}\left(t_{1}-s\right) B x_{p}(s) d s \| \\
\quad \widetilde{J}_{m} J_{m} \| \int_{0}^{t_{2}} t_{2}^{1-\eta}\left(t_{2}-s\right)^{v(1-\zeta)-1} s^{\zeta-1} Q_{\zeta}(s)\left[\phi(0)-F_{1}(0, \phi(0))\right] d s \\
\Gamma(1-\zeta)) \\
-\int_{0}^{t_{1}} t_{1}^{1-\eta}\left(t_{1}-s\right)^{v(1-\zeta)-1} s^{\zeta-1}\left[\phi(0)-F_{1}(0, \phi(0))\right] d s \|
\end{aligned}
$$




$$
\begin{aligned}
& +M_{0} \widetilde{J}_{m}\left\|t_{2}^{1-\eta} A^{\mu} F_{1}\left(t_{2}, p_{t_{2}}+\hat{\beta}_{t_{2}}\right)-t_{1}^{1-\eta} A^{\mu} F_{1}\left(t_{1}, p_{t_{1}}+\hat{\beta}_{t_{1}}\right)\right\| \\
& +t_{2}^{1-\eta} \widetilde{J}_{m}\left\|\int_{t_{1}}^{t_{2}}\left(t_{2}-s\right)^{\zeta-1} A^{1-\mu} \mathscr{S}_{\zeta}\left(t_{2}-s\right) A^{\mu} J^{-1} F_{1}\left(s, p_{s}+\hat{\beta}_{s}\right) d s\right\| \\
& +\widetilde{J}_{m} \| \int_{0}^{t_{1}}\left[t_{2}^{1-\eta}\left(t_{2}-s\right)^{\zeta-1}-t_{1}^{1-\eta}\left(t_{1}-s\right)^{\zeta-1}\right] A^{1-\mu} \mathscr{S}_{\zeta}\left(t_{2}-s\right) \\
& (\times) A^{\mu} J^{-1} F_{1}\left(s, p_{s}+\hat{\beta}_{s}\right) d s \| \\
& +t_{1}^{1-\eta} \widetilde{J}_{m} \| \int_{0}^{t_{1}-\delta}\left(t_{1}-s\right)^{\zeta-1} \\
& (\times)\left[A^{1-\mu} \mathscr{S}_{\zeta}\left(t_{2}-s\right)-A^{1-\mu} \mathscr{S}_{\zeta}\left(t_{1}-s\right)\right] A^{\mu} J^{-1} F_{1}\left(s, p_{s}+\hat{\beta}_{s}\right) d s \| \\
& +t_{1}^{1-\eta} \widetilde{J}_{m} \| \int_{t_{1}-\delta}^{t_{1}}\left(t_{1}-s\right)^{\zeta-1}\left[A^{1-\mu} \mathscr{S}_{\zeta}\left(t_{2}-s\right)-A^{1-\mu} \mathscr{S}_{\zeta}\left(t_{1}-s\right)\right] \\
& (\times) A^{\mu} J^{-1} F_{1}\left(s, p_{s}+\hat{\beta}_{s}\right) d s \| \\
& +t_{2}^{1-\eta} \widetilde{J}_{m}\left\|\int_{t_{1}}^{t_{2}}\left(t_{2}-s\right)^{\zeta-1} \mathscr{S}_{\zeta}\left(t_{2}-s\right) F_{2}\left(s, p_{s}+\hat{\beta}_{s}, \int_{0}^{s} e\left(s, \tau, p_{\tau}+\hat{\beta}_{\tau}\right) d \tau\right) d s\right\| \\
& +\widetilde{J}_{m} \int_{0}^{t_{1}} \|\left[t_{2}^{1-\eta}\left(t_{2}-s\right)^{\zeta-1}-t_{1}^{1-\eta}\left(t_{1}-s\right)^{\zeta-1}\right] \mathscr{S}_{\zeta}\left(t_{2}-s\right) \\
& (\times) F_{2}\left(s, p_{s}+\hat{\beta}_{s}, \int_{0}^{s} e\left(s, \tau, p_{\tau}+\hat{\beta}_{\tau}\right) d \tau, \int_{0}^{c} f\left(s, \tau, p_{\tau}+\hat{\beta}_{\tau}\right) d \tau\right) d s \| \\
& +t_{1}^{1-\eta} \widetilde{J}_{m} \| \int_{0}^{t_{1}-\delta}\left(t_{1}-s\right)^{\zeta-1}\left[\mathscr{S}_{\zeta}\left(t_{2}-s\right)-\mathscr{S}_{\zeta}\left(t_{1}-s\right)\right] \\
& (\times) F_{2}\left(s, p_{s}+\hat{\beta}_{s}, \int_{0}^{s} e\left(s, \tau, p_{\tau}+\hat{\beta}_{\tau}\right) d \tau, \int_{0}^{c} f\left(s, \tau, p_{\tau}+\hat{\beta}_{\tau}\right) d \tau\right) d s \| \\
& +t_{1}^{1-\eta} \widetilde{J}_{m} \| \int_{t_{1}-\delta}^{t_{1}}\left(t_{1}-s\right)^{\zeta-1}\left[\mathscr{S}_{\zeta}\left(t_{2}-s\right)-\mathscr{S}_{\zeta}\left(t_{1}-s\right)\right] \\
& (\times) F_{2}\left(s, p_{s}+\hat{\beta}_{s}, \int_{0}^{s} e\left(s, \tau, p_{\tau}+\hat{\beta}_{\tau}\right) d \tau, \int_{0}^{c} f\left(s, \tau, p_{\tau}+\hat{\beta}_{\tau}\right) d \tau\right) d s \| \\
& +t_{2}^{1-\eta} \widetilde{J}_{m}\left\|\int_{t_{1}}^{t_{2}}\left(t_{2}-s\right)^{\zeta-1} \mathscr{S}_{\zeta}\left(t_{2}-s\right) B x_{p}(s) d s\right\| \\
& +\widetilde{J}_{m} \int_{0}^{t_{1}}\left\|\left[t_{2}^{1-\eta}\left(t_{2}-s\right)^{\zeta-1}-t_{1}^{1-\eta}\left(t_{1}-s\right)^{\zeta-1}\right] \mathscr{S}_{\zeta}\left(t_{2}-s\right) B x_{p}(s) d s\right\| \\
& +t_{1}^{1-\eta} \widetilde{J}_{m}\left\|\int_{0}^{t_{1}-\delta}\left(t_{1}-s\right)^{\zeta-1}\left[\mathscr{S}_{\zeta}\left(t_{2}-s\right)-\mathscr{S}_{\zeta}\left(t_{1}-s\right)\right] B x_{p}(s) d s\right\| \\
& +t_{1}^{1-\eta} \widetilde{J}_{m}\left\|\int_{t_{1}-\delta}^{t_{1}}\left(t_{1}-s\right)^{\zeta-1}\left[\mathscr{S}_{\zeta}\left(t_{2}-s\right)-\mathscr{S}_{\zeta}\left(t_{1}-s\right)\right] B x_{p}(s) d s\right\| \\
& \leq \sum_{i=1}^{16} T_{i}
\end{aligned}
$$

where

$$
\begin{aligned}
& T_{1}=\frac{M \widetilde{J}_{m} J_{m}}{\Gamma(\nu(1-\zeta)) \Gamma(\zeta)}\left\|\int_{t_{1}}^{t_{2}} t_{2}^{1-\eta}\left(t_{2}-s\right)^{v(1-\zeta)-s_{s}{ }^{-1}}\left[\phi(0)-F_{1}(0, \phi(0))\right] d s\right\|, \\
& T_{2}=t_{2}^{1-\eta} \frac{M \widetilde{J}_{m} J_{m}}{\Gamma(\zeta)}\left\|\int_{0}^{t_{1}}\left[\left(t_{2}-s\right)^{\zeta-1}-\left(t_{1}-s\right)^{\zeta-1}\right] s^{\zeta-1}\left[\phi(0)-F_{1}(0, \phi(0))\right] d s\right\|, \\
& T_{3}=\left(t_{2}^{1-\eta}-t_{1}^{1-\eta}\right) \frac{M \widetilde{J}_{m} J_{m}}{\Gamma(\zeta)}\left\|\int_{0}^{t_{1}}\left(t_{1}-s\right)^{\zeta-1} s^{\zeta-1}\left[\phi(0)-F_{1}(0, \phi(0))\right] d s\right\|, \\
& T_{4}=M_{0} \widetilde{J}_{m}\left[t_{2}^{1-\eta}-t_{1}^{1-\eta}\right] M_{g}^{\prime}\left(1+r^{\prime}\right), \\
& T_{5}=t_{2}^{1-\eta} \frac{K_{1-\mu} \Gamma(1+\mu)}{\Gamma(1+\zeta \mu)}\left[\left(t_{2}-t_{1}\right)^{\zeta \mu}\right] \widetilde{J}_{m} M_{g}^{\prime}\left(1+r^{\prime}\right),
\end{aligned}
$$




$$
\begin{aligned}
& T_{6}=\frac{\zeta K_{1-\mu} \Gamma(1+\mu)}{\Gamma(1+\zeta \mu)} \widetilde{J}_{m} M_{g}^{\prime}\left(1+r^{\prime}\right) \| \\
& (\times) \int_{0}^{t_{1}} \frac{1}{\left(t_{2}-s\right)^{\zeta(1-\mu)}}\left[t_{2}^{1-\eta}\left(t_{2}-s\right)^{\zeta-1}-t_{1}^{1-\eta}\left(t_{1}-s\right)^{\zeta-1}\right] d s \|, \\
& T_{7}=t_{1}^{1-\eta} \frac{\left(t_{1}\right)^{\zeta}-(\delta)^{\zeta}}{\zeta} \sup _{\left[0, t_{1}-\delta\right]}\left[A^{1-\mu} \mathscr{S}_{\zeta}\left(t_{2}-s\right)-A^{1-\mu} \mathscr{S}_{\zeta}\left(t_{1}-s\right)\right] \widetilde{J}_{m} M_{g}^{\prime}\left(1+r^{\prime}\right), \\
& T_{8}=t_{1}^{1-\eta} \frac{\delta^{\zeta}}{\zeta} \sup _{\left[0, t_{1}-\delta\right]}\left[A^{1-\mu} \mathscr{S}_{\zeta}\left(t_{2}-s\right)-A^{1-\mu} \mathscr{S}_{\zeta}\left(t_{1}-s\right)\right] \widetilde{J}_{m} M_{g}^{\prime}\left(1+r^{\prime}\right) \\
& T_{9}=\frac{M \widetilde{J}_{m} c^{1-\eta}}{\Gamma(\zeta)} \frac{\left(t_{2}-t_{1}\right)^{(1+K)(1-\rho)}}{(1+K)^{(1-\rho)}}\left\|\epsilon_{1}\right\|_{L^{\frac{1}{\rho}}} \mathrm{Y}\left(r^{\prime}+c E_{0}\left(1+r^{\prime}\right)+c E_{1}\left(1+r^{\prime}\right)\right), \\
& T_{10}=\frac{M \widetilde{J}_{m} \mathrm{Y}\left(r^{\prime}+c E_{0}\left(1+r^{\prime}\right)\right)}{\Gamma(\zeta)}\left\|\int_{0}^{t_{1}}\left[t_{2}^{1-\eta}\left(t_{2}-s\right)^{\zeta-1}-t_{1}^{1-\eta}\left(t_{1}-s\right)^{\zeta-1}\right] \epsilon_{1}(s) d s\right\|, \\
& T_{11}=c^{1-\eta} \widetilde{J}_{m} \frac{\left(t_{1}^{(1+K)}-\delta^{(1+K)}\right)^{1-\rho}}{(1+K)^{1-\rho}} \sup _{\left[0, t_{1}-\delta\right]}\left\|\mathscr{S}_{\zeta}\left(t_{2}-s\right)-\mathscr{S}_{\zeta}\left(t_{1}-s\right)\right\|\left\|\epsilon_{1}\right\|_{L^{\frac{1}{\rho}}} \\
& (\times) \mathrm{Y}\left(r^{\prime}+c E_{0}\left(1+r^{\prime}\right)+c E_{1}\left(1+r^{\prime}\right)\right), \\
& T_{12}=t_{1}^{1-\eta} \frac{\delta^{(1+K)(1-\rho)}}{(1+K)^{1-\rho}} \frac{2 M \widetilde{J}_{m}}{\Gamma(\zeta)}\left\|\epsilon_{1}\right\|_{L^{\frac{1}{\rho}}} \mathrm{Y}\left(r^{\prime}+c E_{0}\left(1+r^{\prime}\right)+c E_{1}\left(1+r^{\prime}\right)\right), \\
& T_{13}=t_{1}^{1-\eta} \frac{M \widetilde{J}_{m} \epsilon_{b}}{\Gamma(\zeta)}\left\|\int_{t_{1}}^{t_{2}}\left(t_{2}-s\right)^{\zeta-1} w(s) d s\right\| \\
& T_{14}=\frac{M \widetilde{J}_{m} \epsilon_{b}}{\Gamma(\zeta)}\left\|\int_{0}^{t_{1}}\left[t_{2}^{1-\eta}\left(t_{2}-s\right)^{\zeta-1}-t_{1}^{1-\eta}\left(t_{1}-s\right)^{\zeta-1}\right] w(s) d s\right\|, \\
& T_{15}=d^{1-\eta} \frac{M \widetilde{J}_{m} \epsilon_{b}}{\Gamma(\zeta)}\left\|\int_{0}^{t_{1}-\delta}\left(t_{1}-s\right)^{\zeta-1} w(s) d s\right\| \sup _{\left[0, t_{1}-\delta\right]}\left\|Q_{\zeta}\left(t_{2}-s\right)-Q_{\zeta}\left(t_{1}-s\right)\right\|, \\
& T_{16}=d^{1-\eta} \frac{M \widetilde{J}_{m} \epsilon_{b}}{\Gamma(\zeta)}\left\|\int_{t_{1}-\delta}^{t_{1}}\left(t_{1}-s\right)^{\zeta-1} w(s) d s\right\| \text {. }
\end{aligned}
$$

The right-hand side of $T_{1}$ to $T_{12}$ tends to ' 0 ' as $\delta \rightarrow 0$. On implementing the absolute continuity of the Lebesgue integral dominance convergence theorem for inequality, we conclude that $T_{1}$ to $T_{12}$ gives ' 0 ' when $t_{2}-t_{1} \rightarrow 0$. Therefore, $\widetilde{Y}\left(F_{r}\right)$ is equicontinuous on $I$.

Step 4: Now, we need to prove that the Mönch's condition holds.

Consider $\left(p^{0}+\hat{\beta}\right)(t)=t^{1-\eta} \mathscr{P}_{\nu, \zeta}(t) \phi(0)$, for all $t \in I$ and $\left(p^{m+1}+\hat{\beta}\right)=\widetilde{Y}\left(p^{m}+\right.$ $\hat{\beta}), m=0,1,2,3, \cdots$ and $\widetilde{Y}$ is relatively compact.

Consider $\mathscr{H} \subseteq F_{r}$ is countable and $\mathscr{H} \subseteq \operatorname{conv}(\{0\} \cup \widetilde{\mathrm{Y}}(\mathscr{H}))$. We need to verify that $\mathscr{T}(\mathscr{H})=0$, where $\mathscr{T}$ is the Hausdorff measure of noncompactness. Consider $\mathscr{H}=$ $\left\{\left(p^{m}+\hat{\beta}\right)\right\}_{m=1}^{\infty}$. Presently we have to verify that $\widetilde{Y}(\mathscr{H})(t)$ is relatively compact in $X$, for all $t \in I$. By referring Theorem 1 ,

$$
\begin{aligned}
\mathscr{T}(\mathscr{H}(t)) & =\mathscr{T}\left(\left\{\left(p^{m}+\hat{\beta}\right)(t)\right\}_{m=0}^{\infty}\right)=\mathscr{T}\left(\left\{\left(p^{0}+\hat{\beta}\right)(t)\right\} \cup\left\{\left(p^{m}+\hat{\beta}\right)(t)\right\}_{m=1}^{\infty}\right) \\
& =\mathscr{T}\left(\left\{\left(p^{m}+\hat{\beta}\right)(t)\right\}_{m=1}^{\infty}\right),
\end{aligned}
$$

and

$$
\begin{aligned}
\mathscr{T}\left(\left\{\left(\widetilde{\mathrm{Y}} p^{m}\right)(t)\right\}_{m=1}^{\infty}\right)= & \mathscr{T}\left(\left\{t^{1-\eta} \int_{0}^{t}(t-s)^{\zeta-1} J^{-1} \mathscr{S}_{\zeta}(t-s)\left[\mathcal{E}_{m}(s)+B x_{p^{m}}(s)\right]\right\}_{m=1}^{\infty}\right) \\
\leq & \frac{2 M \widetilde{J}_{m} c^{1-\eta}}{\Gamma(\zeta)} \int_{0}^{t}(t-s)^{\zeta-1} \mathscr{T}\left(\left\{\mathcal{E}_{m}(s)\right\}_{m=1}^{\infty}\right) d s \\
& +\frac{2 M \widetilde{J}_{m} c^{1-\eta}}{\Gamma(\zeta)} \int_{0}^{t}(t-s)^{\zeta-1} \mathscr{T}\left(\left\{B x_{p^{m}}(s)\right\}_{m=1}^{\infty}\right) d s
\end{aligned}
$$




$$
=U_{1}+U_{2}
$$

Now,

$$
\begin{aligned}
U_{1} \leq & \frac{2 M \widetilde{J}_{m} c^{1-\eta}}{\Gamma(\zeta)} \int_{0}^{t}(t-s)^{\zeta-1} \mathscr{T}\left(\left\{\mathcal{E}_{m}\left(p_{s}^{m}+\hat{\beta}_{s}\right)\right\}_{m=1}^{\infty}\right) d s \\
\leq & \frac{2 M \widetilde{J}_{m} c^{1-\eta}}{\Gamma(\zeta)} \int_{0}^{t}(t-s)^{\zeta-1}\left[\mathscr{T}\left(\left\{p^{m}(\tau+\varphi)+\hat{\beta}(\tau+\varphi)\right\}_{m=1}^{\infty}\right)\right. \\
& \left.+\mathscr{T}\left(\left\{\int_{0}^{\tau} e\left(\tau, \varepsilon, p_{\varepsilon}^{m}+\hat{\beta}_{\varepsilon}\right) d \varepsilon, \int_{0}^{b} f\left(\tau, \varepsilon, p_{\varepsilon}^{m}+\hat{\beta}_{\varepsilon}\right) d \varepsilon\right\}_{m=1}^{\infty}\right)\right] d s \\
\leq & \frac{2 M \widetilde{J}_{m} c^{1-\eta}}{\Gamma(\zeta)} K_{2}\left(1+2 \epsilon_{3}^{*}+2 \epsilon_{4}^{*}\right) \sup _{-\infty<\varphi \leq 0} \mathscr{T}(\mathscr{X}(\varphi)), \\
U_{2}= & \mathscr{T}\left(\left\{c^{1-\eta} \int_{0}^{t}(t-s)^{\eta-1} J^{-1} \mathscr{S}_{\zeta}(c-s) B w_{p^{m}}(s) d s\right\}_{m=1}^{\infty}\right) \\
\leq & \frac{2 M \widetilde{J}_{m} \epsilon_{b} c^{1-\eta}}{\Gamma(\zeta)} \int_{0}^{t}(t-s)^{\eta-1} \mathscr{T}\left(\left\{w_{p^{m}}(s)\right\}_{m=1}^{\infty}\right) d s \\
\leq & \frac{2 M \widetilde{J}_{m} \epsilon_{b} c^{1-\eta}}{\Gamma(\zeta)^{2}} \int_{0}^{t}(t-s)^{\eta-1} \quad \\
& (\times) \mathscr{T}\left[W^{-1}\left(\int_{0}^{c}(c-s)^{\eta-1} J^{-1} \mathscr{S}_{\zeta}(c-s) \mathscr{T}\left(E_{m}\left(p_{s}^{m}+\hat{\phi}_{s}\right)_{m=1}^{\infty}\right) d s\right)\right] d s \\
\leq & \frac{2 M \widetilde{J}_{m} \epsilon_{b} c^{1-\eta}}{\Gamma(\zeta)} \int_{0}^{t}(t-s)^{\eta-1} M_{\mathscr{W}}(s) d s \frac{2 M}{\Gamma(\zeta)} K_{2} \widetilde{J}_{m}\left(1+2 \epsilon_{3}^{*}+2 \epsilon_{4}^{*}\right) \quad \sup _{-\infty<\varphi \leq 0} \mathscr{T}(\mathscr{X}(\varphi)) \\
\leq & \frac{4 M^{2} \widetilde{J}_{m} \epsilon_{b} c^{1-\eta}}{\Gamma(\zeta)^{2}} K_{0} K_{2} \widetilde{J}_{m}\left(1+2 \epsilon_{3}^{*}+2 \epsilon_{4}^{*}\right) \sup _{-\infty<\varphi \leq 0} \mathscr{T}(\mathscr{X}(\varphi)) .
\end{aligned}
$$

Now

$$
U_{1}+U_{2} \leq \frac{2 M \widetilde{J}_{m} K_{2}\left(1+2 \epsilon_{3}^{*}+2 \epsilon_{4}^{*}\right) c^{1-\eta}}{\Gamma(\zeta)}\left[1+\frac{2 M \widetilde{J}_{m} K_{0} \epsilon_{b}}{\Gamma(\zeta)}\right] \sup _{-\infty<\varphi \leq 0} \mathscr{R}(\mathscr{X}(\varphi)) .
$$

By referring Lemma 5,

$$
\mathscr{T}(\widetilde{Y}(H)) \leq L^{*} \mathscr{T}(H) .
$$

Hence by using Mönch's condition, we have

$$
\mathscr{T}(\widetilde{\mathrm{Y}}) \leq \mathscr{T}\left(\operatorname{conv}(\{0\} \cup(\widetilde{\mathrm{Y}}(H)))=\mathscr{T}(\widetilde{\mathrm{Y}}(H)) \leq L^{*} \mathscr{T}(H),\right.
$$

this implies $\mathscr{T}(H)=0$. Therefore, $\widetilde{Y}$ has a fixed point $y \in F_{r}$, in view of Lemma 6. Next, $u=p+\hat{\beta}$ is a mild solution of the fractional system (1)-(2) satisfying $u(c)=u^{1}$, then the fractional system (1)-(2) is controllable on $X$.

\section{Nonlocal Conditions}

Physical problems prompted the development of evolution equations with nonlocal conditions. In [54,55], the authors explored nonlocal issues for the first time in 1990, obtaining the existence and uniqueness of mild solutions for nonlocal differential equations of integer order. For more details on the systems with integer of fractional orders, one can refer $[29,30,32,35,45,54,55]$. Assume that nonlocal Hilfer fractional delay Volterra-Fredholm integro-differential system has the following form

$$
D_{0^{+}}^{v, \zeta}\left[J u(t)-F_{1}\left(t, u_{t}\right)\right]=A u(t)+B x(t)
$$




$$
\begin{gathered}
+F_{2}\left(t, u_{t}, \int_{0}^{t} e\left(t, \tau, u_{\tau}\right) d \tau, \int_{0}^{c} f\left(t, \tau, u_{\tau}\right) d \tau\right), \quad t \in I=(0, c], \\
I_{0^{+}}^{(1-v)(1-\zeta)} u(0)=\phi+g\left(u_{t_{1}}, u_{t_{2}}, u_{t_{3}}, \cdots, u_{t_{n}}\right) \in \mathcal{R}_{l},
\end{gathered}
$$

where $0<t_{1}<t_{2}<t_{3}<\cdots<t_{n} \leq d, g: \mathcal{R}_{l}^{n} \rightarrow \mathcal{R}_{l}$ and satisfies the following: $\left(\mathbf{H}_{\mathbf{6}}\right) g: \mathcal{R}^{n} \rightarrow \mathcal{R}$ is continuous, there exists $K_{i}(g)>0$ such that

$$
\left\|g\left(u_{1}, u_{2}, \cdots, u_{n}\right)-g\left(w_{1}, w_{2}, \cdots, w_{n}\right)\right\| \leq \sum_{i=1}^{n} K_{i}(g)\left\|u_{i}-w_{i}\right\|_{\mathcal{R}_{l^{\prime}}}
$$

for all $u_{i}, w_{i} \in \mathcal{R}_{l}$ and consider $K_{g}=\sup \left\{\left\|g\left(u_{1}, u_{2}, \cdots, u_{n}\right)\right\|: u_{i} \in \mathcal{R}_{l}\right\}$.

Definition 10. A function $u:(-\infty, c] \rightarrow X$ is said to be a mild solution of (9) and (10) if $\left.u_{0}=\phi+g\left(u_{t_{1}}, u_{t_{2}}, u_{t_{3}}, \cdots, u_{t_{n}}\right)(0)\right) \in \mathcal{R}_{l}$ on $(-\infty, 0]$ and

$$
\begin{aligned}
u(t)=J^{-1} & \mathscr{P}_{v, \zeta}(t) J\left[\phi(0)-F_{1}\left(0, \phi(0)+g\left(u_{t_{1}}, u_{t_{2}}, u_{t_{3}}, \cdots, u_{t_{n}}\right)(0)\right)\right]+J^{-1} F_{1}\left(t, u_{t}\right) \\
& +\int_{0}^{t}(t-s)^{\zeta-1} J^{-1} A \mathscr{S}_{\zeta}(t-s) J^{-1} F_{1}\left(s, u_{s}\right) d s \\
& +\int_{0}^{t}(t-s)^{\zeta-1} J^{-1} \mathscr{S}_{\zeta}(t-s) F_{2}\left(s, u_{s}, \int_{0}^{s} e\left(s, \tau, u_{\tau}\right) d \tau, \int_{0}^{c} f\left(s, \tau, u_{\tau}\right) d \tau\right) d s \\
& +\int_{0}^{t}(t-s)^{\zeta-1} J^{-1} \mathscr{S}_{\zeta}(t-s) B x(s) d s, t \in I,
\end{aligned}
$$

is satisfied.

Theorem 3. Assume that the hypotheses $\left(\mathbf{H}_{\mathbf{0}}\right)-\left(\mathbf{H}_{\mathbf{6}}\right)$ are satisfied, then the fractional system (9) and (10) is controllable if

$$
L^{*}=\frac{2 M \widetilde{J}_{m} K_{2}\left(1+2 \epsilon_{3}^{*}+2 \epsilon_{4}^{*}\right) c^{1-\eta}}{\Gamma(\zeta)}\left[1+\frac{2 M \widetilde{J}_{m} K_{0} \epsilon_{b}}{\Gamma(\zeta)}\right], \text { for some } \frac{1}{2}<\zeta<1 .
$$

\section{Examples}

\subsection{Abstract System}

Assume that the Hilfer fractional differential system with control of the following form

$$
\begin{aligned}
D_{0^{+}}^{v, \frac{2}{3}}[u(s, \hbar) & \left.-\frac{\partial^{2}}{\partial \hbar^{2}} u(s, \hbar)+\int_{0}^{\pi} \zeta(z, \hbar) u(s, z) d z\right]=\frac{\partial^{2}}{\partial \hbar^{2}} u(s, \hbar) \\
& +E\left(s, \int_{-\infty}^{s} \varrho_{1}(\varepsilon-s) u(\varepsilon, \hbar) d \varepsilon, \int_{0}^{\theta} \int_{-\infty}^{0} \xi_{2}(\tau, x, \varepsilon-\tau) u(\varepsilon, \hbar) d \varepsilon d \tau,\right. \\
& \left.\int_{0}^{c} \int_{-\infty}^{0} \xi_{3}(\tau, x, \varepsilon-\tau) u(\varepsilon, \hbar) d \varepsilon d \tau\right)+\mathscr{V}(s, \hbar), \quad \tau \in[0, \pi], s \in[0, c], \\
& \left.I^{(1-v) \frac{1}{3}}[u(s, \hbar)]\right|_{\hbar=0}=u_{0}(\hbar), \hbar \in[0, \pi], \\
& u(s, 0)=u(s, \pi)=0, s \geq 0, \\
& u(0, \mu)=s(\mu), 0 \leq \mu \leq \pi,
\end{aligned}
$$

where $D_{0^{+}}^{v, \frac{2}{3}}$ denotes the Hilfer fractional derivative of order $\left(\zeta=\frac{2}{3}\right)$ and type $v, \mathscr{V}$ : $I \times[0,1] \times[0,1] \times \mathbb{R} \rightarrow \mathbb{R}$ is continuous.

To transform the fractional system (11)-(14) to abstract form, assume $X=L^{2}(0, \pi)$ and $A: D(A) \subset X \rightarrow X, J: D(J) \subset X \rightarrow X$ be defined by $A w=w^{\prime \prime}$, and $P w=w-A$ where $D(A)$ and $D(J)$ is given by $\left\{w \in U: w, w^{\prime}\right.$ are absolutely continuous, $\left.w(0)=w(\pi)=0\right\}$. Then, $A$ and $J$ are presented as $A w=\sum_{m=1}^{\infty} n^{2}\left\langle w, z_{m}\right\rangle z_{m}, w \in D(A), J w=\sum_{m=1}^{\infty}(1+$ 
$\left.m^{2}\right)\left\langle w, z_{m}\right\rangle z_{m}, w \in D(J)$ where $z_{m}(x)=\sqrt{\frac{2}{\pi}} \sin (m x), m=1,2,3, \cdots$ are the orthonormal of vectors of $A$. Then, for $z \in U$, we have

$$
\begin{gathered}
P^{-1} z=\sum_{m=1}^{\infty} \frac{1}{\left(1+m^{2}\right)}\left\langle z, z_{m}\right\rangle z_{m}, \\
A J^{-1} z=\sum_{m=1}^{\infty} \frac{m^{2}}{\left(1+m^{2}\right)}\left\langle z, z_{m}\right\rangle z_{m},
\end{gathered}
$$

and

$$
Q_{\mu}(\theta) z=\sum_{m=1}^{\infty} \exp \left(\frac{m^{2} \theta}{1+m^{2}}\right)\left\langle z, z_{m}\right\rangle z_{m} .
$$

$A$ is defined by $T(\theta) z(\tau)=z(\theta+\tau)$ for $z \in X, T(\theta)$ is not compact on $X$ with $\mathscr{T}(T(\theta) D) \leq$ $\mathscr{T}(D), \mathscr{T}$ is the Hausdorff measure of noncompactness.

Here $A$ is an infinitesimal generator of a semigroup $\{T(s), s \geq 0\}$ in $X$ and which is presented as $T(s) w(\varepsilon)=w(s+\varepsilon)$, for $w \in U, T(s)$ is not compact on $X$ with $\mathscr{T}(T(s) D) \leq$ $\mathscr{T}(D)$. Furthermore, $s \rightarrow w\left(s^{\frac{2}{3}}+\varepsilon\right) u$ is equicontinuous ([45]), where $s \geq 0$ and $\hbar \in(0, \infty)$. We assume $F_{1}:[0, \pi] \times X \rightarrow X$ and $F_{2}:[0, \pi] \times X \times X \times X \rightarrow X$ by

$$
\begin{aligned}
& e(\theta, s)=\int_{-\infty}^{\theta} \xi_{2}(\theta, \tau, \varepsilon) s(\varepsilon) d \varepsilon, \\
& f(\theta, s)=\int_{-\infty}^{c} \xi_{3}(\theta, \tau, \varepsilon) s(\varepsilon) d \varepsilon, \\
& F_{1}(u)(\varepsilon)=\int_{0}^{\pi} \zeta(z, \varepsilon) u(s, \varepsilon) d \varepsilon, \\
& F_{2}\left(\theta, s, \int_{0}^{\theta} e(\varepsilon, s) d \varepsilon, \int_{0}^{c} f(\varepsilon, s) d \varepsilon\right) \\
&=E\left(s, \int_{-\infty}^{s} v_{1}(\varepsilon-s) u(\varepsilon, \zeta) d \varepsilon, \int_{0}^{\epsilon} e(\varepsilon, s)(\tau) d \varepsilon, \int_{0}^{c} f(\varepsilon, s)(\tau) d \varepsilon\right),
\end{aligned}
$$

and $D_{0^{+}}^{v, \frac{2}{3}}(u)(s)(\mu)=\frac{\partial^{\frac{2}{3}}}{\partial \mu^{\frac{2}{3}}} u(s, \mu), u(s)(\varepsilon)=u(s, \varepsilon)$.

Let $B: X \rightarrow X$ be given by $(B x)(s)(\hbar)=\mathscr{V} x(s, \hbar), 0<s<1$. For $\hbar \in(0, \pi), W$ is given by

$$
W x(\hbar)=\int_{0}^{1}(1-s)^{\frac{-1}{3}} \mathscr{S}_{\zeta}(1-s) \mathscr{V} x(s, \hbar) d s
$$

where

$$
\mathscr{S}_{\frac{2}{3}}=\frac{2}{3} \int_{0}^{\infty} \hbar \xi_{\frac{2}{3}}(\hbar) x\left(t^{\frac{2}{3}} \hbar\right) d \hbar,
$$

and for $\hbar \in(0, \infty)$

$$
\begin{gathered}
\xi_{\frac{2}{3}}(\hbar)=\frac{3}{2} \hbar^{-1-\frac{-5}{2}} \bar{x}_{\frac{2}{3}}\left(\hbar^{\frac{-3}{2}}\right), \\
\bar{x}_{\frac{2}{3}}(\hbar)=\frac{1}{\pi} \sum_{n=1}^{\infty}(-1)^{n-1} t^{-\frac{2}{3} n-1} \frac{\Gamma\left(\frac{2}{3} n+1\right)}{n !} \sin \left(\frac{2 n \pi}{3}\right),
\end{gathered}
$$

where $\xi_{\frac{2}{3}}$ is determined on $(0, \infty)$, i.e.,

$$
\xi_{\frac{2}{3}}(\hbar) \geq 0, \quad \hbar \in(0, \infty) \quad \text { and } \quad \int_{0}^{\infty} \xi_{\frac{2}{3}}(\hbar) d \hbar=1
$$


Finally $F_{1}, F_{2}$ and $\mathscr{V}$ satisfy the hypotheses $\left(\mathbf{H}_{\mathbf{1}}\right)-\left(\mathbf{H}_{\mathbf{5}}\right)$, thus all the requirements of the Theorem 2 are satisfied, then the fractional system (11)-(14) is controllable on $I$.

\subsection{Filter System}

An advanced filter is a framework that performs mathematical operations on an inspected, digitized sign to decrease or upgrade certain highlights of the prepared signal. Propelled by the plans examined in $[13,19,29,56,57]$, we presented a filter design for our framework which is shown in Figure 1. Figure 1 portrays the rough pattern of block diagram which helps to improve the viability of arrangement with least measure of sources of input and which is presented as follows.

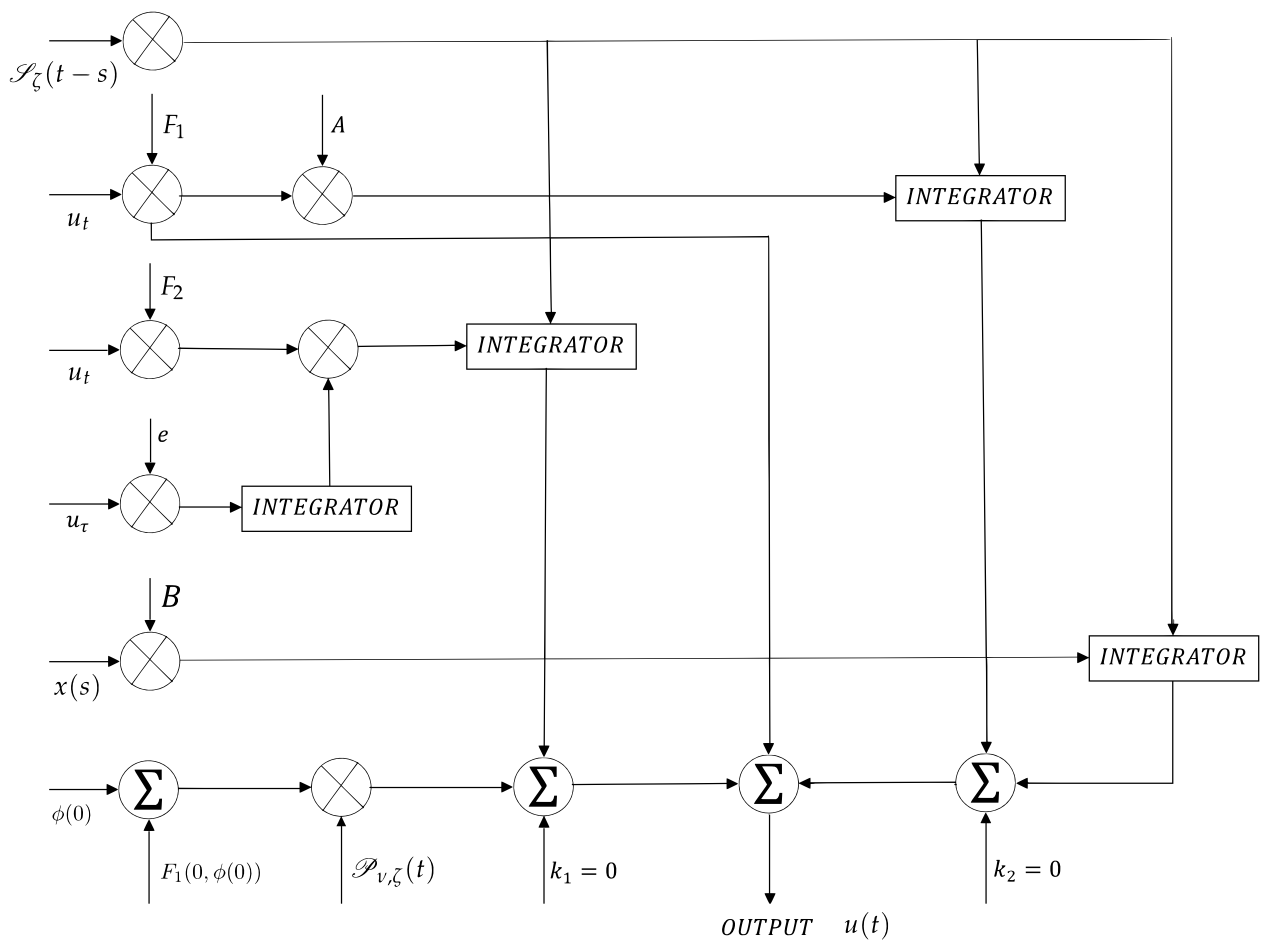

Figure 1. Filter System.

Product modulator $(\mathrm{PM})-1$ receives inputs $u_{t}$ and $F_{1}$ generates the output as $F_{1}\left(t, u_{t}\right)$. PM-2 receives $A$ and $F_{1}\left(t, u_{t}\right)$ generates $A F_{1}\left(t, u_{t}\right)$. PM-4 receives $u_{\tau}$ and $f$ generates $f\left(t, \tau, u_{\tau}\right)$. PM-4 receives $u_{t}$ and $F_{2}$ produces $F_{2}\left(t, u_{t}\right)$. PM-5 receives $u_{\tau}$ and $e$ generates $e\left(t, \tau, u_{\tau}\right)$. PM-6 receives $F_{2}\left(t, u_{t}\right), e\left(t, \tau, u_{\tau}\right)$ and $f\left(t, \tau, u_{\tau}\right)$ generates

$$
F_{2}\left(t, u_{t}, \int_{0}^{t} e\left(t, \tau, u_{\tau}\right) d \tau, \int_{0}^{c} f\left(t, \tau, u_{\tau}\right) d \tau\right)
$$

PM-7 receives $x(t)$ and $B$ generates $B x(t)$. PM-8 receives $\left[\phi(0)-F_{1}(0, \phi(0))\right]$ and $\mathscr{P}_{v, \zeta}(t)$ at time $t=0$, generates $\mathscr{P}_{v, \zeta}(t)$. The integrators execute the integral of

$$
\mathscr{S}_{\zeta}(t)\left[A \mathscr{S}_{\zeta}(t) F_{1}\left(t, u_{t}\right)+F_{2}\left(t, u_{t}, \int_{0}^{t} e\left(t, \tau, u_{\tau}\right) d \tau, \int_{0}^{c} f\left(t, \tau, u_{\tau}\right) d \tau\right)+B x(t)\right] \text {, }
$$

over $t$.

Additionally, Inputs $\mathscr{S}_{\zeta}(t), A F_{1}\left(t, u_{t}\right)$ are joined and multiplying with the output on the interval $(0, t) . \mathscr{S}_{\zeta}(t), F_{2}\left(t, u_{t}, \int_{0}^{t} e\left(t, \tau, u_{\tau}\right) d \tau, \int_{0}^{c} f\left(t, \tau, u_{\tau}\right) d \tau\right)$ are joined and multiplying with the output on the interval $(0, t) . \mathscr{S}_{\zeta}(t), B x(t)$ are joined and multiplying with the output on the interval $(0, t)$.

Finally, if we shift all the outputs from the integrators to summer network, then, the output of $u(t)$ is achieved, which is bounded and controllable. 


\section{Conclusions}

The exact controllability of Sobolev-type Hilfer fractional neutral integro-differential systems via measure of noncompactness is the topic of our article. The main conclusions of our paper are based on theoretical ideas such as fractional calculus, the measure of noncompactness, and the fixed-point approach. First, we looked at the exact controllability of mild solutions for fractional evolution systems. Then we expanded on our findings to consider the system in nonlocal conditions. Finally, we presented theoretical and practical applications to aid in the efficacy of the discussion.

Author Contributions: Conceptualization, V.V., K.S.N.; methodology, V.V., K.S.N.; software, V.V., S.F.A. and K.S.N.; formal analysis, V.V., S.F.A. and K.S.N.; investigation, V.V., S.F.A. and K.S.N.; resources, V.V., S.F.A. and K.S.N.; writing - original draft preparation, V.V., S.F.A. and K.S.N.; writingreview and editing, V.V., S.F.A. and K.S.N.; visualization, V.V., S.F.A. and K.S.N.; supervision, V.V., S.F.A. and K.S.N.; project administration, V.V., S.F.A. and K.S.N.; funding acquisition, V.V., S.F.A. and K.S.N. All authors have read and agreed to the published version of the manuscript.

Funding: This research received no external funding.

Institutional Review Board Statement: Not applicable.

Informed Consent Statement: Not applicable.

Data Availability Statement: Not applicable.

Conflicts of Interest: The authors declare no conflict of interest.

\section{References}

1. Baleanu, D.; Diethelm, K.; Scalas, E.; Trujillo, J.J. Fractional Calculus Models and Numerical Methods; Series on Complexity, Nonlinearity and Chaos; World Scientific Publishing: Boston, MA, USA, 2012.

2. Baleanu, D.; Machado, J.A.T.; Luo, A.C.J. Fractional Dynamics and Control; Springer: New York, NY, USA.: 2012. [CrossRef]

3. Baleanu, D.; Gunvenc, Z.B.; Tenreiro Machado, J.A. New Trends in Nanotechnology and Fractional Calculus Applications; Springer: Dordrecht, The Netherlands, 2010. [CrossRef]

4. Kilbas, A.; Srivastava, H.; Trujillo, J.J. Theory and Applications of Fractional Differential Equations; Elsevier: Amesterdam, The Netherlands, 2006.

5. Lakshmikantham, V.; Vatsala, A.S. Basic theory of fractional differential equations. Nonlinear Anal. 2008, 69, 2677-2682. [CrossRef]

6. Lakshmikantham, V.; Leela, S.; Devi, J.V. Theory of Fractional Dynamic Systems; Cambridge Scientific Publishers: Cambridge, UK, 2009.

7. Podlubny, I. Fractional Differential Equations, An Introduction to Fractional Derivatives, Fractional Differential Equations, to Method of Their Solution and Some of Their Applications; Academic Press: San Diego, CA, USA, 1999.

8. Zhou, Y. Fractional Evolution Equations and Inclusions: Analysis and Control; Elsevier: New York, NY, USA, 2015.

9. Zhou, Y. Basic Theory of Fractional Differential Equations; World Scientific: Singapore, 2014.

10. Demir, D.D.; Bildik, N.; Sinir, B.G. Application of fractional calculus in the dynamics of beams. Bound. Value Probl. 2012, 135, 1687-2770.

11. El-sayed, A.M.A. Fractional order diffusion wave equation. Int. J. Theor. Phys. 1966, 35, 311-322. [CrossRef]

12. Vijayakumar, V. Approximate controllability results for abstract neutral integro-differential inclusions with infinite delay in Hilbert spaces. IMA J. Math. Control. Inf. 2018, 35, 297-314. [CrossRef]

13. Vijayakumar, V.; Udhayakumar, R. A new exploration on existence of Sobolev-type Hilfer fractional neutral integro-differential equations with infinite delay. Numer. Methods Partial. Differ. Equ. 2021, 37, 750-766. [CrossRef]

14. Craciun, E.M.; Sadowski, T.; Rabaea, A. Stress concentration in an anisotropic body with three equal collinear cracks in Mode II of fracture. I. Analytical study. Zamm-Z. Fur Angew. Math. Und Mech. 2014, 94, 721-729. [CrossRef]

15. Othman, I.A.M.; Said, S.; Marin, M. A novel model of plane waves of two-temperature fiber-reinforced thermoelastic medium under the effect of gravity with three-phase-lag model. Int. J. Numer. Methods Heat Fluid Flow 2019, 29, 4788-4806. [CrossRef]

16. Vlase, S.; Teodorescu, P.P.; Itu, C.; Scutaru, M.L. Elasto-dynamics of a solid with a general "rigid" motion using FEM model Part I, Theoretical approach. Rom. J. Phys. 2013, 58, 872-881.

17. Agarwal, S.; Bahuguna, D. Existence of solutions to Sobolev-type partial neutral differential equations. J. Appl. Math. Stoch. Anal. 2006, 2006, 16308. [CrossRef]

18. Kavitha, K.; Vijayakumar, V.; Udhayakumar, R.; Sakthivel, N.; Nisar, K.S. A note on approximate controllability of the Hilfer fractional neutral differential inclusions with infinite delay. Math. Methods Appl. Sci. 2021, 44, 4428-4447. [CrossRef]

19. Ravichandran, C.; Valliammal, N.; Nieto, J.J. New results on exact controllability of a class of fractional neutral integro-differential systems with state-dependent delay in Banach spaces. J. Frankl. Inst. 2019, 356, 1535-1565. [CrossRef] 
20. Zhou, Y.; Jiao, F. Existence of mild solutions for fractional neutral evolution equations. Comput. Math. Appl. 2010, 59, 1063-1077. [CrossRef]

21. Zhou, Y.; Jiao, F.; Li, J. Existence and uniqueness for fractional neutral differential equations with infinite delay. Nonlinear Anal. 2009, 71, 3249-3256. [CrossRef]

22. Hilfer, R. Application of Fractional Calculus in Physics; World Scientific: Singapore, 2000.

23. Hilfer, R. Experimental evidence for fractional time evolution in glass materials. Chem. Phys. 2002, 284, 399-408. [CrossRef]

24. Gu, H.; Trujillo, J. Existence of mild solution for evolution equation with Hilfer fractional derivative. Appl. Math. Comput. 2015, 257, 344-354. [CrossRef]

25. Dineshkumar, C.; Udhayakumar, R.; Vijayakumar, V.; Nisar, K.S. A discussion on the approximate controllability of Hilfer fractional neutral stochastic integro-differential systems. Chaos Solitons Fractals 2020. [CrossRef]

26. Kavitha, K.; Vijayakumar, V.; Udhayakumar, R.; Nisar, K.S. Results on the existence of Hilfer fractional neutral evolution equations with infinite delay via measures of noncompactness. Math. Methods Appl. Sci. 2021, 44, 1438-1455. [CrossRef]

27. Kavitha, K.; Vijayakumar, V.; Udhayakumar, R.; Ravichandran, C. Results on controllability of Hilfer fractional differential equations with infinite delay via measures of noncompactness. Asian J. Control 2021. [CrossRef]

28. Nisar, K.S.; Vijayakumar, V. Results concerning to approximate controllability of non-densely defined Sobolev-type Hilfer fractional neutral delay differential system. Math. Methods Appl. Sci. 2021, 44, 13615-13632. [CrossRef]

29. Subashini, R.; Jothimani, K.; Nisar, K.S.; Ravichandran, C. New results on nonlocal functional integro-differential equations via Hilfer fractional derivative. Alex. Eng. J. 2020, 59, 2891-2899. [CrossRef]

30. Yang, M.; Wang, Q. Approximate controllability of Hilfer fractional differential inclusions with nonlocal conditions. Math. Methods Appl. Sci. 2017, 40, 1126-1138. [CrossRef]

31. Dineshkumar, C.; Udhayakumar, R.; Vijayakumar, V.; Nisar, K.S.; Shukla, A. A note on the approximate controllability of Sobolev type fractional stochastic integro-differential delay inclusions with order $1<r<2$. Math. Comput. Simul. 2021, 190, 1003-1026.

32. Wang, J.R.; Zhang, Y.R. Nonlocal initial value problems for differential equation with Hilfer fractional derivative. Appl. Math Comput. 2015, 266, 850-859. [CrossRef]

33. Chang, Y.K.; Li, W.T. Controllability of Sobolev type semilinear functional differential and integrodifferential inclusions with an unbounded delay. Georgian Math. J. 2006, 13, 11-24. [CrossRef]

34. Feckan, M.; Wang, J.; Zhou, Y. Controllability of fractional functional evolution equations of Sobolev type via characteristic solution operators. J. Optim. Theory Appl. 2013, 156, 79-95. [CrossRef]

35. Ji, S.; Li, G.; Wang, M. Controllability of impulsive differential systems with nonlocal conditions. Appl. Math. Comput. 2011, 217, 6981-6989. [CrossRef]

36. Raja, M.M.; Vijayakumar, V.; Udhayakumar, R.; Zhou, Y. A new approach on the approximate controllability of fractional differential evolution equations of order $1<r<2$ in Hilbert spaces. Chaos Solitons Fractals 2020, 141, 110310.

37. Mohan Raja, M.; Vijayakumar, V.; Udhayakumar, R. A new approach on approximate controllability of fractional evolution inclusions of order $1<r<2$ with infinite delay. Chaos Solitons Fractals 2020, 141, 110343.

38. Ravichandran, C.; Baleanu, D. Existence results for fractional neutral functional integro-differential evolution equations with infinite delay in Banach spaces. Adv. Differ. Equ. 2013, 2013, 215. [CrossRef]

39. Shukla, A.; Sukavanam, N.; Pandey, D.N. Approximate controllability of fractional semilinear control system of order $\alpha \in(1,2]$ in Hilbert spaces. Nonlinear Stud. 2015, 22, 131-138.

40. Shukla, A.; Sukavanam, N.; Pandey, D.N. Approximate Controllability of semilinear fractional control systems of order $\alpha \in(1,2]$ with infinite delay. Mediterr. J. Math. 2016, 13, 2539-2550. [CrossRef]

41. Shukla, A.; Sukavanam, N.; Pandey, D.N. Approximate controllability of fractional semilinear stochastic system of order $\alpha \in(1,2]$. J. Dyn. Control. Syst. 2017, 23, 679-691. [CrossRef]

42. Shukla, A.; Sukavanam, N.; Pandey, D.N. Approximate controllability of semilinear fractional stochastic control system. Asian-Eur. J. Math. 2018, 11, 1850088. [CrossRef]

43. Vijayakumar, V.; Ravichandran, C.; Murugesu, R. Nonlocal controllability of mixed Volterra-Fredholm type fractional semilinear integro-differential inclusions in Banach spaces. Dyn. Contin. Discret. Impuls. Syst. Ser. Appl. Algorithms 2013, 20, 485-502.

44. Wang, J.R.; Feckan, M.; Zhou, Y. Controllability of Sobolev type fractional evolution systems. Dyn. Partial. Differ. Equ. 2014, 11, 71-87. [CrossRef]

45. Wang, J.R.; Fan, Z.; Zhou, Y. Nonlocal controllability of semilinear dynamic systems with fractional derivative in Banach spaces. J. Optim. Theory Appl. 2012, 154, 292-302. [CrossRef]

46. Pazy, A. Semilgroups of Linear Operators and Applications to Partial Differential Equations; Springer: New York, NY, USA, 1983.

47. Lightbourne, J.H.; Rankin, S. A partial functional differential equation of Sobolev type. J. Math. Anal. Appl. 1983, 93, 328-337. [CrossRef]

48. Yan, B. Boundary value problems on the half-line with impulses and infinite delay. J. Math. Anal. Appl. 2001, 259, 94-114. [CrossRef]

49. Banas, J.; Goebel, K. Measure of Noncompactness in Banach Spaces. In Lecture Notes in Pure and Applied Matyenath; Marcel Dekker: New York, NY, USA, 1980.

50. Deimling, K. Multivalued Differential Equations; De Gruyter: Berlin, Germany, 1992. 
51. Kamenskii, M.; Obukhovskii, V.; Zecca, P. Condensing Multivalued Maps and Semilinear Differential Inclusions in Banach Spaces;De Gruyter: Berlin, Germany, 2001. [CrossRef]

52. O'Regan, D.; Precup, R. Existence criteria for integral equations in Banach spaces. J. Inequalities Appl. 2001, 6, 77-97. [CrossRef]

53. M'onch, H. Boundary value problems for nonlinear ordinary differential equations of second order in Banach spaces. Nonlinear Anal. 1980, 4, 985-999. [CrossRef]

54. Byszewski, L. Theorems about the existence and uniqueness of solutions of a semilinear evolution nonlocal Cauchy problem. J. Math. Anal. Appl. 1991, 162, 494-505. [CrossRef]

55. Byszewski, L.; Akca, H. On a mild solution of a semilinear functional-differential evolution nonlocal problem. J. Appl. Math. Stoch. Anal. 1997, 10, 265-271. [CrossRef]

56. Chandra, A.; Chattopadhyay, S. Design of hardware efficient FIR filter: A review of the state of the art approaches. Eng. Sci. Technol. Int. J. 2016, 19, 212-226. [CrossRef]

57. Zahoor, S.; Naseem, S. Design and implementation of an efficient FIR digital filter. Cogent Eng. 2017, 4, 1323373. [CrossRef] 